1 Loss of E-cadherin Induces IGF1R Activation Revealing a Targetable Pathway in Invasive

\title{
2 Lobular Breast Carcinoma
}

3 Ashuvinee Elangovan ${ }^{1,2}$, Jagmohan $\operatorname{Hooda}^{2}$, Laura Savariau ${ }^{2,3}$, Susrutha

4 Puthanmadhomnarayanan ${ }^{2}$, Megan E. Yates ${ }^{2,4}$, Jian Chen $^{2}$, Daniel D. Brown ${ }^{5}$, Priscilla F.

5 McAuliffe ${ }^{2,6}$, Steffi Oesterreich ${ }^{2,7}$, Jennifer M. Atkinson ${ }^{2,7, *}$, Adrian V. Lee ${ }^{2,7, *}$

$7 \quad{ }^{1}$ Molecular Genetics and Developmental Biology Graduate Program, University of Pittsburgh

8 School of Medicine, Pittsburgh PA. ${ }^{2}$ Women's Cancer Research Center, University of Pittsburgh

9 Medical Center (UPMC) Hillman Cancer Center (HCC), Magee-Womens Research Institute,

10 Pittsburgh, PA. ${ }^{3}$ Department of Human Genetics, University of Pittsburgh Graduate School of

11 Public Health, Pittsburgh, PA, ${ }^{4}$ Medical Scientist Training Program, University of Pittsburgh

12 School of Medicine, Pittsburgh, PA. ${ }^{5}$ Institute for Precision Medicine, Pittsburgh, PA.

$13{ }^{6}$ Department of Surgery, Division of Surgical Oncology, Section of Breast Surgery, University of

14 Pittsburgh School of Medicine, Pittsburgh, PA. ${ }^{7}$ Department of Pharmacology \& Chemical

15 Biology, University of Pittsburgh, Pittsburgh, PA.

17 Running Title: Loss of $C D H 1$ sensitizes cells to IGF pathway activation.

Keywords: Invasive lobular carcinoma, E-cadherin, IGF, receptor availability, patient-derived

19 organoids 
20 Financial support: Research funding for this project was provided in part by Susan G. Komen

21 Scholar awards (SAC110021 to AVL and SAC160073 to SO), the Breast Cancer Research

22 Foundation (to AVL and SO), and the National Cancer Institute (R01CA224909).

24 *Corresponding Authors:

25 Adrian V. Lee, $\mathrm{PhD}$

26 leeav@upmc.edu

27 Phone: 4126417724, Fax: 4126416456

28 Women's Cancer Research Center, UPMC Hillman Cancer Center

29204 Craft Avenue

30 Pittsburgh, PA 15213, USA

31

32 Jennifer M. Atkinson, $\mathrm{PhD}$

33 xavierjm@upmc.edu

34 Phone: 4126417724 , Fax: 4126416456

35 Women's Cancer Research Center, UPMC Hillman Cancer Center

36204 Craft Avenue

37 Pittsburgh, PA 15213, USA

38

39

40 Conflict of Interest Disclosure: The authors declare no potential conflicts of interest.

41 Word count: 5273 words

42 Total number of figures: 7 main figures, 6 supplemental figures and 1 supplemental table 


\section{Abstract}

44 No Special Type breast cancer (NST; commonly known as Invasive Ductal Carcinoma (IDC))

45 and Invasive Lobular Carcinoma (ILC) are the two major histological subtypes of breast cancer with significant differences in clinicopathological and molecular characteristics. The defining

47 pathognomonic feature of ILC is loss of cellular adhesion protein, E-cadherin $(C D H 1)$. We have previously shown that E-cadherin functions as a negative regulator of the Insulin-like Growth Factor 1 Receptor (IGF1R) and propose that E-cadherin loss in ILC sensitizes cells to growth

50 factor signaling which thus alters their sensitivity to growth factor signaling inhibitors and their

51 downstream activators. To investigate this potential therapeutic vulnerability, we generated

52 CRISPR-mediated $C D H 1$ knockout (CDH1 KO) IDC cell lines (MCF7, T47D, ZR75.1) to uncover the mechanism by which loss of E-cadherin results in IGF pathway activation. CDH1 KO cells demonstrated enhanced invasion and migration that was further elevated in response to

55 IGF1, serum and Collagen I. $C D H 1 \mathrm{KO}$ cells exhibited increased sensitivity to IGF resulting in

56 elevated downstream signaling. Despite minimal differences in membranous IGF1R levels

57 between wildtype (WT) and $C D H 1 \mathrm{KO}$ cells, significantly higher ligand-receptor interaction was observed in the $C D H 1 \mathrm{KO}$ cells, potentially conferring enhanced downstream signaling activation. Critically, increased sensitivity to IGF1R, PI3K, Akt and MEK inhibitors was observed in $C D H 1 \mathrm{KO}$ cells and ILC patient-derived organoids, suggesting that these targets require further exploration in ILC treatment and that $C D H 1$ loss may be exploited as a biomarker of response for patient stratification. 


\section{Introduction}

67 Despite significant diagnostic and therapeutic advancements, breast cancer remains a leading 68 cause of malignancy associated mortality for women worldwide. Breast cancer is a heterogenous 69 disease that can be categorized based on its histopathology and molecular features. The major 70 histological subtypes of breast cancer are No Special Type (NST) which is often referred to as

71 Invasive Ductal Carcinoma (IDC: $\sim 80 \%$ of all cases) and Invasive Lobular Carcinoma (ILC:

$72 \sim 10-15 \%$ of all cases) (1-3). Although IDC and ILC are clinically managed as the same disease,

73 there is an increasing appreciation of their differences in terms of molecular alterations,

74 pathology, and prognosis (4-7). The diminished prevalence of ILC consequently has resulted in

75 this subtype being historically understudied and therefore implores the need to better characterize

76 the disease to establish precision medicine approaches to treatment $(4,8)$. ILC tumors are largely

77 characterized by their growth in a single file, discohesive manner with presence of stromal

78 infiltration $(5-7,9)$. Approximately $95 \%$ of ILC cases present with loss of $C D H 1$, with the

79 majority due to genomic alterations such as loss of heterozygosity and/or truncating frameshift

80 mutations $(6,10,11)$. In addition, other molecular alterations such as $P I K 3 C A$ activation, PTEN

81 loss, and mutations in TBX3 and FOXA1 are detected at higher levels in ILC tumors compared

82 to IDC tumors $(4,12)$. Enhanced Akt signaling has also been observed in several ILC cell line 83 and mouse models (13).

As one of the activators of Akt, the Insulin-like Growth Factor 1 (IGF1) pathway has long been

86 associated with breast cancer progression (14-18). IGF1 is an essential component in cellular

87 proliferation and survival $(15,19)$. Binding of IGF1 to its receptor (IGF1R) or the insulin

88 receptor (IR) stimulates autophosphorylation and activation of the PI3K and MAPK pathways 
among others $(15,18,20)$. Due to high levels of circulating IGF1 being associated with poor

90

91

92

93

94

95

96

97

98

99

100

101

\section{2}

103

prognosis in breast cancer (21), it is imperative to understand the dysregulation of the IGF1

pathway in tumors. Numerous IGF1R inhibitors have been evaluated in preclinical and clinical settings, including humanized monoclonal antibodies that downregulate the receptor and small molecule tyrosine kinase inhibitors which inhibit pathway activation (20, 22-26). Unfortunately, these trials demonstrated little to no response in the recruited broad patient populations despite promising preclinical efficacy $(13,27-31)$, suggesting the necessity to better stratify patients to ensure efficient validation of therapies while maximizing response metrics.

To that end, we recently reported E-cadherin as a modulator of IGF1 signaling and a potential biomarker of inhibitor response (32). Briefly, transient E-cadherin knockdown sensitized cell lines to IGF1 signaling and to IGF1R inhibition $(32,33)$, and we further reported that ILC tumors have higher IGF1/2 expression and pIGF1R/IR activation compared to IDC tumors (13, 33). In this present study, we have expanded upon our earlier observations by generating CRISPR-mediated $C D H 1$ knockout ( $C D H 1 \mathrm{KO}$ ) IDC cell lines to elucidate the role of Ecadherin individually in more detail. Here we report that loss of E-cadherin renders cells sensitive to IGF1, IGF2 and insulin signaling by increasing IGF1R receptor availability for ligand binding resulting in enhanced cell migration/invasion and contextual increases in sensitivity to IGF1R, PI3K, Akt and MEK inhibitors. Given previous evidence for E-cadherin regulation of other growth factor pathways $(13,30)$, we also extended our analyses to additional growth factor signaling pathways. Our findings provide insights into better understanding the mechanics of E-cadherin function as well as the potential for a targeted therapeutic intervention in ILC patient populations. 


\section{Materials and Methods}

\section{Cell culture}

114 Cell lines utilized in this study were obtained from ATCC: MCF7 (RRID: CVCL_0031), T47D

115 (RRID: CVCL_0553), ZR75.1 (RRID: CVCL_0588), MDA-MB-134-VI (RRID: CVCL_0617),

116 MDA-MB-231 (RRID: CVCL_0062) and Asterand for SUM44PE (RRID: CVCL_3424). Cell

117 lines were maintained in 10\% fetal bovine serum (FBS; Life Technologies) supplemented media

118 (Thermo Fisher Scientific): MDA-MB-134 in 1:1 DMEM: L-15; MCF7 and MDA-MB-231 in

119 DMEM; and T47D and ZR75.1 in RPMI. SUM44PE was maintained in DMEM/F12 with 2\%

120 charcoal stripped serum (CSS; Life Technologies) with additional supplements as previously

121 described (5). Cell lines were cultured for less than 6 months at a time, routinely tested to be

122 Mycoplasma free and authenticated by the University of Arizona Genetics Core (Tucson,

123 Arizona) by short tandem repeat DNA profiling.

\section{CDH1 knockout cell line generation}

CRISPR mediated knockout of $C D H 1$ in MCF7 and T47D cells was performed by utilizing the

127 Gene Knockout Kit (V1) from Synthego (Redwood City, California) as previously described (31, 128 34). The parental cell line was used a comparator for MCF7 and T47D, referred as wildtype

129 (WT). For ZR75.1, a doxycycline inducible Cas9 was utilized harboring either short guide RNA

130 (sgRNA) for $C D H 1$ or a non-targeting control (NTC) sequence and was introduced via an

131 adenoviral vector. For ZR75.1, a total of 3 rounds of infection and Puromycin selection was

132 performed before single cell sorting. 8 clones from each KO and NTC cells were isolated by 133 single cell cloning and combined to generate a pool for subsequent experiments. 


\section{Haptotaxis, migration and invasion assays}

136

137

138

139

140

141

142

143

144

145

146

147

148

149

150

151

152

153

154

155 156 immunoblotting (30).

For haptotaxis experiments, the QCM Haptotaxis Cell Migration Assay Collagen I (EMD

Millipore \#ECM582) kit was used as previously described (8). For Transwell migration assays, transparent 24 well PET membranes of $8 \mu \mathrm{m}$ pore size (Fisher Scientific \# 08-771-21) were used. For Collagen I invasion assays, QCM Collagen Cell Invasion Assay (EMD Millipore \#ECM551) was used according to manufacturer's protocol. For the latter two assays, cells were plated at a density of 300,000 cells/well in $300 \mu \mathrm{L} 0.5 \%$ FBS media in the top chamber; all bottom chambers were filled with $0.5 \%$ FBS media +/- 5nM IGF1 (GroPep Bioreagents \# AQU100) or full serum $(10 \%)$ media. Cells were incubated at $37^{\circ} \mathrm{C}$ for 72 hours. Excess cells were removed from the top chambers using cotton swabs and inserts were stained with Crystal Violet (Sigma-Aldrich \#C0775) before being imaged on an Olympus SZX16 dissecting microscope and quantified with ImageJ software. Quantifications were normalized to low serum WT samples and p-values calculated with one-way ANOVA.

\section{Receptor availability assay}

Cells were seeded in $6 \mathrm{~cm}$ plates (Fisher \#08-772-E), and serum starved overnight after achieving a $70-80 \%$ confluency. Cells were then stimulated with biotinylated IGF1 (GroPep \#AQU100) for 15 minutes at $4^{\circ} \mathrm{C}$ to reduce receptor internalization. Following PBS washes on ice, cells were treated with 2mM BS3 crosslinker (Thermo Scientific \#21580) reconstituted in PBS (pH 8.0) with $6 \mathrm{mM} \mathrm{KCl}$ and $10 \mathrm{mM}$ EGTA for 1 hour at $4^{\circ} \mathrm{C}$ with occasional rocking. BS3 quenching was performed with $10 \mathrm{mM}$ Glycine for 15 minutes and cells were harvested for subsequent 


\section{Dose Response assays}

159 Cells were plated in $50 \mu \mathrm{L}$ of media at 9,000 cells/well in 2D and ULA (Corning \#3474) 96-well plates. Treatments were added 24 hours post seeding in an additional $50 \mu \mathrm{L}$ of respective media.

IGF1R inhibitors BMS-754807 (Selleckchem \#S1124) and OSI-906 (Selleckchem \#S1091), dissolved in DMSO with a final $\leq 0.5 \%$ DMSO concentration in treatments. Plates were collected at day 6 and measured by CellTiter-Glo (Promega \#PR-G7573) following the manufacturer's

166 protocol. Cell viability values were analyzed following blank cell deductions and normalization

167 to vehicle readings. IC50 values for viability were calculated by nonlinear regression and 168 statistical differences evaluated using sum-of-squares Global f-test $(\mathrm{p}<0.05)$. Synergy was assessed using SynergyFinder (35).

\section{In silico analysis}

172 Gene expression data from the Sweden Cancerome Analysis Network-Breast (SCAN-B) study 173 was downloaded from Gene Expression Omnibus, accession GSE96058 (36). Differential gene 174 expression between luminal A lobular $(\mathrm{N}=265)$ and ductal $(\mathrm{N}=1165)$ breast cancer samples

175 was assessed using DESeq2 (37). Tumor purity for SCAN-B samples was estimated using the R 176 package ESTIMATE (37). An FDR cut-off of 0.05 was used to identify significantly

177 differentially expressed genes. Heatmap for genes of interest was created using the R package 178 ComplexHeatmap. Gene set variation analysis (GSVA) was performed on log transformed gene 179 FPKM matrices using the R package GSVA (38) with default parameter "Gaussian” for kernel 
180 selection. Gene sets of interest were obtained from MSigDB version 7.4. The GSVA enrichment

181 scores were compared between luminal A lobular and ductal breast cancer samples using Mann-

182 Whitney U test. Gene set enrichment analysis (GSEA version 4.1.0. Broad Institute) (39) was

183 also conducted on normalized raw counts with default parameters and customized gene sets of

184 interest obtained from MSigDB.

186 Organoid culture

187 IDC organoids (IPM-BO-56 and 102) and ILC organoids (IPM-BO-30, 41, 46, 77 and 114) were 188 established by the Institute for Precision Medicine (Pittsburgh, PA) and maintained in media as 189 detailed in (40) with 1nM $\beta$-estradiol (Sigma-Aldrich \#E8875) supplementation. For dose 190 response assays, organoids were dissociated to single cell suspension with Trypsin and plated in $19150 \mu \mathrm{L}$ of organoid media at 3,000 or 5,000 cells/well in 96-well round bottom plates (Falcon 192 \#353227) depending on the growth rate. Akt inhibitor MK2206 (Selleckchem \#S1078; dissolved 193 in DMSO) treatments were added 24 hours post seeding in an additional $50 \mu \mathrm{L}$ of organoid 194 media. Organoids were monitored every day to ensure vehicle treated wells were growing well 195 and remained at an appropriate density. Media was refreshed on day 6 and plates collected on 196 day 12 with cell viability quantified by CellTiter-Glo 3D (Promega \#G9681). Dose response 197 assay analysis was performed as described above. 
202

All data generated in this study are available within the article and its supplementary files. SCAN-B data analyzed in this study was obtained from Gene Expression Omnibus (GEO) at GSE96058.

\section{Results}

\section{IGF pathway activity is enhanced in ILC patient samples and cell lines in vitro}

Analysis of the publicly available Sweden Cancerome Analysis Network-Breast (SCAN-B) study allowed a direct comparison of growth factor signaling differences between luminal A IDC $(\mathrm{n}=1165)$ and luminal A ILC $(\mathrm{n}=265)$ tumors. Significantly higher IGF1 and IGF2 expression were observed in ILC tumors in addition to several other growth factor signaling related genes (Fig 1A). PI3K/Akt signaling was significantly higher in ILC by gene set enrichment analysis (GSEA) (Fig 1B) and gene set variation analysis (GSVA) (Fig 1C). IGF1/2 signaling activation was also higher in ILC (Fig 1D), strengthening our hypothesis regarding IGF pathway activation in ILC over IDC tumors.

We assessed the differences in IGF pathway activity in IDC (MCF7, T47D, ZR75.1) and ILC (MDA-MB-134-VI, SUM44PE) cell lines. Both ILC cell lines showed higher pIGF1R/IR expression after IGF1 stimulation compared to the IDC cell lines, though the expression of total IGF1R/IR varied across cell models (Fig 1E). pAkt (S473) levels, as a measure of downstream pathway activation, did not always correlate with pIGF1R/IR levels. We observed Akt activation in the absence of ligands in T47D and ZR75.1 cells, while MCF7 cells demonstrated robust pAkt induction despite modest pIGF1R/IR levels. These results are not entirely surprising given the existing PI3K activating mutations in MCF7 and T47D (41-43), and PTEN loss in ZR75.1 cells 
222 (43) which may contribute to the Akt activation. We further assessed the effects of insulin and

223 again observed that ILC cell lines demonstrated enhanced activation of pIGF1R/IR, although

224 with this ligand ZR75.1 also showed robust activation (Supp Fig 1A). To test whether there was

225 a difference between ILC and IDC cell lines in response to ligands targeting other cell surface

226 receptors that may be regulated by E-cadherin $(30,44-46)$, we assessed the effects of EGF

227 stimulation in our panel of cell lines (Supp Fig 1B). Little EGFR activation could be detected in

228 ILC cell lines suggesting cellular context specific changes in growth factor signaling activation.

229 Taken together these data support increased IGF pathway activity in ILC compared to IDC in

230 both representative patient tumors and in a cell line models.

CDH1 knockout cells as a model to study the role of E-cadherin in regulating the IGF pathway

234 As previously reported (32, 33), and expanded herein (Fig. 1), E-cadherin negatively regulates

235 IGF pathway activity. To better understand this regulation, we generated CRISPR-mediated

236 CDH1 knockout (CDH1 KO) IDC cell lines of MCF7, T47D and ZR75.1. Both MCF7 and T47D

237 demonstrate successful and complete knockout of $C D H 1$ while ZR75.1 CDH1 KO cells retained

238 some expression of E-cadherin though markedly reduced compared to WT cells (Fig $\mathbf{2 A}, \mathbf{B})$.

239 ZR75.1 cells harbor copy number amplification of $C D H 1$ which we believe made complete

240 knockout of $C D H 1$ in this model challenging $(42,47)$.

241 We first assessed cell morphology given the known role of E-cadherin in formation of cell-cell

242 junctions. As shown in Fig 2C, MCF7 and T47D CDH1 KO cells have a rounded morphology

243 compared to their parental (WT) cells in 2D culture. In addition, they also demonstrated 
244 decreased cell-cell attachment as well as decreased cell-plate attachment. In ultra-low attachment

245 (ULA) conditions, MCF7 and T47D CDH1 KO cells appeared to form less tight, more grape-like

246 cell clusters synonymous with ILC cell aggregates $(8,48)$. We did not note any obvious

247 difference in either 2D or ULA conditions between ZR75.1 WT and CDH1 KO cells.

248 Examination of E-cadherin by immunofluorescence confirmed loss in all three cell lines models

249 (Fig 2D). Furthermore, similar to ILC cell lines $(8,49)$, p120 catenin was re-localized to the

250 cytoplasm in the E-cadherin null $\mathrm{CDH} 1 \mathrm{KO}$ cells. Consistent with our earlier observations,

251 ZR75.1 CDH1 KO cells showed residual E-cadherin expression, and p120 expression was

252 visualized in both the cytoplasm and at the cell membrane.

253 As a function of the cadherin complex, E-cadherin stabilizes $\beta$-catenin on the cell membrane and

254 prevents its release into the cytoplasm. The release of $\beta$-catenin results in free $\beta$-catenin being

255 targeted for proteasomal degradation and/or its accumulation resulting in subsequent activation

256 of Wnt signaling. We thus examined expression of $\beta$-catenin following GSK inhibitor (CHIR-

257 99021; Selleckchem \#S2924) treatment. With vehicle treatment, both MCF7 and T47D CDH1

$258 \mathrm{KO}$ cells exhibited little appreciable $\beta$-catenin expression, which was only elevated following

259 24-hour GSK inhibitor treatment (Supp Fig 1C), suggesting that CDH1 KO alone does not alter

$260 \beta$-catenin accumulation. Together, these data support the successful generation of $C D H 1 \mathrm{KO}$

261 IDC cell line models which display phenotypes consistent with loss of E-cadherin functionality

262 including the characteristic re-localization of p120 as seen in human ILC tumors. 


\section{Loss of E-cadherin sensitizes cells to IGF signaling pathway activation}

267 To understand the signaling effects of E-cadherin loss, we subjected WT and CDH1 KO cells to

268 IGF1 stimulation. We observed enhanced sensitivity in all three $C D H 1 \mathrm{KO}$ cell lines by proxy of

269 higher pIGF1R/IR and pAkt activation (Fig 3A). This enhanced signaling response of $C D H 1 \mathrm{KO}$

270 cells to WT cells was evident to the greatest extend upon 5nM IGF1 stimulation: 2.72-fold, 1.3-

271 fold and 1.5-fold higher signaling in MCF7, T47D and ZR74.1 CDH1 KO cells respectively. The

272 pAkt elevation was not apparent in ZR75.1 cells, possibly accounted by PTEN loss in this model

273 as discussed previously. We next assessed the effects of E-cadherin loss on IGF2, and insulin

274 signaling and observed enhanced IGF1R/IR activation in MCF7 and ZR75.1 CDH1 KO cells

275 following ligand stimulation (Fig 3B, C). This enhanced signaling translated into enhanced Akt

276 activation in the ZR75.1 CDH1 KO cells, but not in the MCF7 CDH1 KO cells. T47D CDH1

277 KO cells showed similar sensitivity to both IGF2 and insulin when compared to WT cells,

278 suggesting an IGF1 specific differential signaling response.

279 To explore if our findings were related to a general growth factor sensitivity following loss of E-

280 cadherin, we stimulated cells with EGF but did not observe any consistent differences in pEGFR

281 Tyr1068 activation, or pAkt levels between CDH1 KO and WT cells (Supp Fig 2A). We

282 specifically examined the effects of E-cadherin deletion on FGFR activity given recent studies

283 on the role of FGFR4 as a driver of endocrine resistance (50), particularly in ILC (51). Cells

284 were stimulated with a cocktail of FGF ligands (FGF 1, 2, 4, 6, 8, 17, 19, 21 and 23) resulting in

285 strong enhancement in both raw and normalized pFGFR4 levels in both MCF7 and T47D CDH1

286 KO cells, despite decreased total FGFR4 levels in the CDH1 KO cells (Supp Fig 2B).

287 Surprisingly, while pFGFR4 was elevated in CDH1 KO cells, downstream activators did not

288 display a difference between WT and CDH1 KO cells. Additionally, in both MCF7 and T47D 
CDH1 KO cells, we observed elevated pathway activity that was sustained for up to 4 hours in the MCF7 cells and up to 2 hours in the T47D cells (Fig 3D). These results were replicated for pAkt activation in the MCF7 CDH1 KO cells but not in the T47D CDH1 KO, warranting additional studies on the sustained receptor activity and its effects on downstream signaling response. Results obtained with ZR75.1 cells were not robust (Supp Fig 2C) despite multiple trials. Collectively, these results suggest that there is an enhancement of IGF1 response in IDC cell lines harboring E-cadherin loss under the conditions tested, but that this increased signaling sensitivity may not be generalizable to other cell surface receptors and is mostly applicable to the IGF1 pathway.

\section{Loss of E-cadherin increases IGF1R availability on the membrane to allow ligand binding}

To understand why and how loss of E-cadherin leads to sensitized IGF1R signaling, we first compared the expression of IGF1R in the WT and CDH1 KO cells. Using cell fractionation, minimal differences between the MCF7 and ZR75.1 WT and CDH1 KO cells were noted (Fig 4A). Higher IGF1R expression was observed in both whole cell and membrane fractions of the T47D CDH1 KO cells compared to WT, which was consistent with increased IGF1R mRNA (Supp Fig 3A). We assessed the availability of the receptor for ligand binding using a biotinylated IGF1 ligand, which was crosslinked to its receptor for subsequent complex quantification via $\alpha$-biotin immunoblotting. MCF7, T47D and ZR74.1 CDH1 KO cells showed a 2.1-fold, 4-fold and 1.7-fold higher ligand-receptor complex respectively when compared to their corresponding WT cell line controls (Fig 4B). This finding was stronger in the T47D CDH1 KO cells, likely due to the higher baseline IGF1R expression in these cells. As E-cadherin has previously been reported to create a repressive EGFR complex (30), we examined for the 
312 presence of a physical interaction between IGF1R and E-cadherin on the cell membrane which

313 potentially could physically and spatially prevent ligands from binding IGF1R. An

314 immunoprecipitation performed for both IGF1R and E-cadherin did not reveal co-IP of either

315 protein despite multiple attempts to optimize conditions (Fig 4C). Imaging of IGF1R to assess

316 localization on the membrane showed no significant differences between the WT and CDH1 KO

317 cells (Fig 4D). Our results suggest that although no significant differences in IGF1R expression

318 was observed between WT and $C D H 1 \mathrm{KO}$ cells, increased receptor availability to bind ligand

319 likely leads to the observed elevated signaling induction upon ligand binding.

\section{Loss of E-cadherin enhances anoikis resistance and increases cell survival}

322 We previously showed increased anchorage independent growth in ILC cells compared to IDC

323 (8). In IDC cells with $C D H 1 \mathrm{KO}$, we similarly found an increase in ULA growth compared to

324 WT cells in the MCF7 and T47D models (31) as has also been seen by others (52). ZR75.1 WT

325 and $C D H 1 \mathrm{KO}$ cells did not show any significant differences in either growth condition (Supp

326 Fig 4A). To explore the previously observed ability for MCF7 and T47D CDH1 KO cells to

327 thrive in ULA conditions, we examined anoikis resistance using an Annexin V and Propidium

328 Iodide staining technique followed by flow cytometry after a 3-day culture in 2D or ULA plates.

329 While the percentage of live cells in 2D were similar between WT and CDH1 KO cells, the

330 fraction of live cells in ULA were significantly higher in the T47D CDH1 KO cells (Fig 5A),

331 consistent with anoikis resistance. No major differences were observed between the MCF7 WT

332 and $\mathrm{CDH} 1 \mathrm{KO}$ cells in either condition. 
333 To further explore cell survival, we performed colony formation assays by plating cells at a low density in either full serum (10\% FBS) or low serum $(0.5 \% \mathrm{FBS})$ containing 5nM IGF1 in 2D plates. T47D CDH1 KO cells showed increased clonogenic survival in both conditions (Fig 5B). CDH1 KO cells also demonstrated increased colonies in full serum compared to WT, but with no colonies observed in low serum conditions. MCF7 CDH1 KO cells showed no clear differences in full serum quantifications, however, they formed a greater number of smaller colonies than the WT cells, with similar observations in low serum conditions. Our data support that loss of Ecadherin may play a context-dependent role in anoikis resistance and cell survival.

\section{Loss of E-cadherin increases collagen I haptotaxis, IGF1-driven migration and serum-}

We investigated if deletion of $C D H 1$ in IDC cell lines confers haptotactic migration towards the extracellular matrix (ECM), a phenotype that is pronounced in ILC cell lines (8). We observed a significantly higher migration towards Collagen I by MCF7 and T47D CDH1 KO cells (Fig 6A) compared to their respective WT cells. Multiple studies have reported on E-cadherin loss permitting cell migration and invasion (53-55); conflicting reports on the requirement of Ecadherin for metastasis have also been reported (56). To explore this inconsistency while assessing the importance of IGF1, CDH1 KO cells were subjected to Transwell migration assays with chemotactic low serum $(0.5 \%$ FBS $)$ media $+/-5 \mathrm{nM} \mathrm{IGF1}$ or full serum $(10 \% \mathrm{FBS})$ media. All three $C D H 1 \mathrm{KO}$ cell models showed increased migration towards serum compared to WT

353 cells (Fig 6B, C). Consistent with the IGF1 sensitive nature of these CDH1 KO cell lines, MCF7 354 and T47D CDH1 KO cells also showed significant migration towards IGF1, a phenotype which 355 was successfully halted upon IGF1R/IR inhibitor, BMS-754807 addition (Supp Fig 4B-D). 
Higher migration of T47D CDH1 KO cells without the presence of any chemotactic gradient

highlights the migratory phenotype driven by the independent loss of E-cadherin in this model.

Given the haptotactic migration towards Collagen I and migration towards IGF1 and serum, we hypothesized that $C D H 1 \mathrm{KO}$ cells may also be able to invade through Collagen I (8) towards a gradient of IGF1 and serum. While there was no Collagen I invasion observed with the MCF7 and ZR75.1 cells (Supp Fig 4E), T47D CDH1 KO cells showed a significant Collagen I invasion towards serum (Fig 6D). It is important to note that neither of these three cell lines are invasive by nature and the invasive phenotypes observed in T47D CDH1 $\mathrm{KO}$ cells is promising and requires greater investigation in the context of tumor invasion.

\section{Loss of E-cadherin sensitizes cells to Fulvestrant and IGF1R/PI3K/Akt/MEK inhibitors in}

\section{a context dependent manner}

Motivated by the increased sensitivity of $\mathrm{CDH} 1 \mathrm{KO}$ cells to IGF ligands and the potential for clinical translation, we sought to determine if these cells are also sensitive to IGF1R inhibitors. cells (BMS-754807 and OSI-906) (Fig 7A, B). Both MCF7 and ZR75.1 CDH1 KO cells did not

372 show any significant differences when compared to WT cells (Supp Fig 5A, B) although

373 BMS754807 treatment did inhibit IGF1R and Akt signaling in these cell lines, albeit at a lower

374 efficacy in $C D H 1 \mathrm{KO}$ cells, potentially owing to their increased ligand sensitivity (Supp Fig

375 5C). Given the higher incidence of PIK3CA hotspot mutations and occurrence of $P T E N$ loss in

376 ILC $(4,12,57)$, we examined if the loss of E-cadherin in these IDC cell lines also sensitizes

377 them to PI3K and Akt inhibitors, Alpelisib and MK-2206, respectively. As with the IGF1R

378 inhibitors, only T47D CDH1 KO cells showed increased sensitivity to MK2206 and an overall 
increased sensitivity trend to Alpelisib upon averaging statistical analysis of repeated experiments (Fig 7C, D, Supp Fig 5A, B, D). Next, we assessed if the absence of susceptibility to IGF1R and Akt inhibitors in the MCF7 $\mathrm{CDH} 1 \mathrm{KO}$ cells might be explained by alternative pathways being activated. We performed combination treatments of BMS-754807 with MEK inhibitor, U0126, and observed a strong additive effect where both MCF7 and T47D CDH1 KO cells were more sensitive than their corresponding WT cells (Fig 7E, F). While this was an expected result in the T47D cells, it is interesting to note the additive effect in the MCF7 CDHI $\mathrm{KO}$ cells as this suggests the activation of alternative pathways following IGF1R, PI3K and Akt independent inhibitions. We further assessed the synergy effect utilizing the SynergyFinder platform (35), with the ZIP method as output (Supp Fig. 6A, B). In MCF7 and T47D WT and CDH1 KO cells, synergy scores were between -9.717 to 10.995 , which validates an additive but not synergistic drug combination effect. As over $90 \%$ of ILC tumors are estrogen receptor (ER) positive, we also investigated the combination effects of Akt inhibition with an ER degrader, Fulvestrant, and observed a higher susceptibility in the T47D CDHI KO cells (Fig 7G, Supp Fig 6C), supporting the notion of targeting the IGF pathway concurrently to targeting ER. These results support the study of combinatory therapies in pre-clinical studies to better target the activation of the IGF pathway following E-cadherin loss. Finally, to analyze pathway inhibition in a more physiologically relevant model, we utilized patient derived ILC and IDC breast organoids (PDO) to compare Akt inhibition sensitivity. As with our cell line results, no significant IDC to ILC comparison was observed, however, ILC organoids did demonstrate a stronger trend in sensitivity to treatment (Fig 7H, Supp Fig 6D), strengthening potential translational implications. 


\section{Discussion}

403

404

405

406

407

408

409

410

411

412

413

414

415

416

417

418

419

420

421 Studies have attributed spatial accessibility of IGF1R for ligand binding as a limiting factor in

422 signaling activation $(30,58)$, and consistent with this notion, we found that loss of E-cadherin

423 confers increased IGF ligand binding by IGF1R, and subsequent pathway activation. Despite

424 previous reports on E-cadherin repressing growth factor receptors $(30,33,58)$ and co- 
425 localization (58), we were unable to find an association by co-IP despite numerous attempts,

426 suggesting that this is not the mechanism affecting the availability of IGF1R for ligand binding

427 when E-cadherin is present. The formation of a ternary complex involving E-cadherin, IGF1R

428 and integrins is an ongoing topic of investigation thought to affect cell mobility (59), which

429 could additionally complicate immunoprecipitation. Similar interactions have been reported for

430 E-cadherin and EGFR, although we did not observe an increased sensitivity to the EGF ligand in

431 our $C D H 1 \mathrm{KO}$ cell lines $(30,60)$. We, and others, have shown that downregulation of E-cadherin

432 by growth factors can promote $\operatorname{EMT}(58,61)$; others have additionally demonstrated growth

433 factor signaling regulation by E-cadherin, suggesting that a bidirectional feedback loop may

434 exist. These studies were initially almost exclusively performed in the context of EGFR where

435 multiple groups had shown that E-cadherin inhibits ligand-dependent activation of EGFR

436 signaling $(30,62,63)$. However, a recent study by Teo and colleagues (13) supported our

437 findings by reporting on ILC cell lines generated from a p53-deficient metastatic mouse model

438 exhibiting had PI3K/AKT pathway activation and enhanced sensitivity to pathway inhibition.

440 Due to the function of E-cadherin in maintaining cell-cell adhesion, its loss through EMT or

441 genetic deletion is often thought to correlate with increased tumor invasion and metastasis (62,

442 64). Other studies, however, have suggested a requirement for E-cadherin in metastasis (56).

443 Here we show that $C D H 1 \mathrm{KO}$ cells demonstrate increased migration towards Collagen I (8). The

444 CDH1 KO cells also showed an enhanced migration towards serum and IGF1, with T47D CDH1

445 KO cells showing increased migration even in the absence of a chemoattractant. T47D CDH1

$446 \mathrm{KO}$ also showed invasion through Collagen I, providing additional in vitro evidence supporting

447 that the loss of E-cadherin may enhance metastatic phenotypes. The in vitro nature of these 
448 findings, however, is an interpretation limitation and in vivo experimentation is needed to better understand the role of E-cadherin in metastasis and validate its effect on IGF signaling.

Inhibitors of downstream activators such as PI3K, Akt and MEK have successfully been

452 developed and approved for the clinic. This is paramount in malignancy with a high percentage

453 of mutations leading to PIK3CA activation and PTEN loss such as in ILC (4), and maybe more

454 so important given the high IGF pathway activation found in the SCAN-B dataset. Other studies with CRISPR MCF7 CDH1 KO cells lines have shown that loss of E-cadherin leads to dependency upon ROS1 and enhanced sensitivity to crizotinib (65) for which a clinical trial is currently underway. We report increased sensitivity to IGF1R/PI3K/Akt and MEK inhibitors in T47D CDH1 KO cells and an increased trend in sensitivity to an Akt inhibitor in ILC PDOs 459 compared to IDC PDOs. Given the genetic landscape of MCF7, T47D and ZR75.1 cells, these models are primed for hyperactive Akt signaling, which has been shown to be further enhanced by loss of E-cadherin. The helical domain mutation in MCF7 cells (E545K) is reported to have a more aggressive phenotype over the kinase domain mutation in T47D (H1047R) (66), which may explain the absence of additional sensitivity in MCF7 CDH1 KO cells to compounds tested

464 since the PIK3CA mutation may effectively mask any potential effect from E-cadherin loss. With 465 the possibility of IGF1R inhibitor efficacy being reduced by hyperactive downstream pathways 466 such as MEK as seen in colon carcinoma (67) and the possibilities of downstream activation 467 inhibition being overhauled by hyperactive receptor tyrosine kinases (68), we tested and 468 observed an additive effect of treating cells with an IGF1R inhibitor in combination with a MEK 469 inhibitor. Further, additive effects of combining Akt inhibitors with Fulvestrant was also found to be beneficial in E-cadherin deficient cells, proving novel therapeutical benefits which require 
471 further exploration in vivo. Future studies will need to test the efficacy of inhibitors targeting the

472 IGF pathway comparing cells +/- CDH1 in vivo and investigate the potential of efficiently

473 exploiting E-cadherin as a biomarker of therapeutic response.

475 In conclusion, this study delineates the role of E-cadherin in regulating enhanced IGF signaling,

476 controlling metastatic phenotypes, and identifying use of specific tyrosine kinase inhibitors that

477 can effectively target elevated IGF1 signaling activation. We reveal novel findings of increased

478 receptor availability for ligand binding following the loss of E-cadherin and increased

479 susceptibility to IGF1R/PI3K/Akt and MEK inhibitors upon CDH1 deletion. Our study adds to a

480 growing body of evidence suggesting that $\mathrm{CDH1}$ may represent a biomarker of response to

481 kinase inhibitors and further exemplifies the need for investigation into the clinical translation of

482 these findings. The in vitro nature of our studies in a small panel of cell lines is an important,

483 notable limitation, but notwithstanding, we have robustly revealed important cellular- and

484 contextual-dependent functions of E-cadherin in IGF driven tumor phenotypes. Our findings

485 require translation into in vivo models with the goal of validating E-cadherin loss as a biomarker

486 of growth factor receptor inhibitor response in breast cancer as well as the utilization of E-

487 cadherin expression as a vital patient stratification tool in clinical trials.

491 (A) Gene expression analysis of growth factor signaling related genes comparing Luminal A

492 IDC and ILC with significantly different expression values displayed. (B) GSEA analysis for 
PI3K/Akt showing significantly higher pathway activation in ILC samples obtained from the SCANB dataset. (C, D) GSVA analysis of Luminal A IDC and ILC samples using respective signature sets from MSigDB with significantly higher pathway activation in ILC. (E) IDC cells

496 (MCF7, T47D, ZR75.1) and ILC cells (MDA-MB-134-VI, SUM44PE) were stimulated with 497 doses of IGF1 or IGF2 (0-5nM) for 15 minutes following an overnight serum starvation.

498 IGF1R/IR and Akt signaling was assessed by Western blotting. Phosphorylation levels of IGF1R 499 (phospho-IGF-I Receptor $\beta$ (Tyr1135/1136)/Insulin Receptor $\beta$ (Tyr1150/1151)) and Akt (S473)

500 were quantified on the LiCOr Odyssey CLx Imaging system and normalized to corresponding 501 total protein levels and loading controls. Ligand treated sample values were further normalized to respective cell line vehicle treated samples. Representative experiment shown for all, N=2-3 for

503 each experiment.

IGF pathway (A) Western blotting and (B) qRT-PCR confirms reduction of E-cadherin in CDH1 KO MCF7, T47D and ZR75.1 CRISPR cell lines compared to wildtype (WT) parental cells. Statistical differences evaluated using paired t-test $\left({ }^{*} \mathrm{p}<0.05\right)$. Representative experiment shown, $\mathrm{N}=2$ (each with two biological and 3 technical replicates). (C) Representative brightfield images (10X magnification) of WT and CDH1 KO cell line models plated in 2D and Ultra-low

511 attachment (ULA) plates. (D) E-cadherin (red) and p120 catenin (green) staining of WT and $512 C D H 1 \mathrm{KO}$ cells confirms $C D H 1$ loss and p120 re-localization in $C D H 1 \mathrm{KO}$ cell models by 513 confocal microscopy (60X objective). Inset in the ZR75.1 CDH1 KO panel shows a zoomed in image. Scale bar: $50 \mu \mathrm{m}$. Representative experiment shown for all, $\mathrm{N}=2-3$ for each experiment. 
Figure 3: Loss of E-cadherin sensitizes cells to IGF signaling pathway activation. Cells were serum starved overnight and stimulated with (A) IGF1, (B) IGF2 or (C) Insulin (0-10nM) for 15 minutes. Cells were harvested for Western blot to assess IGF1R/IR and Akt signaling. For quantification, phosphorylated protein levels were normalized to corresponding total protein levels and loading controls. Ligand treated sample values were further normalized to respective

521 cell line vehicle treated samples. (D) Cells were treated with 10nM IGF1 for a time course from 522 0-6 hours to assess the duration of signaling activity between WT and CDH1 KO cells.

523 Representative experiment shown for all, $\mathrm{N}=2-3$ for each experiment.

Figure 4: Loss of E-cadherin increases IGF1R availability on the membrane to allow ligand

527 KO cells to assess whole cell and membrane IGF1R expression levels. IGF1R bands were 528 quantified and normalized to membrane control, AIF1. (B) Cells were stimulated with 529 biotinylated IGF1 $(0-10 \mathrm{nM})$ for 10 minutes and crosslinked to assess ligand-receptor complex 530 levels between WT and CDH1 KO cells. (C) Immunoprecipitation of IGF1R and E-cadherin in 531 T47D cells was assessed for a co-IP of other proteins, with p-120 catenin assessed as a known 532 interactor of E-cadherin. (D) Cell lines were dual stained for EpCAM (green) and IGF1R (red) 533 and imaged by confocal microscopy at a $60 \mathrm{X}$ objective. Scale bar: $50 \mu \mathrm{m} .12$ images for each cell

534 line were quantified and graphed. Representative experiment shown for all, $\mathrm{N}=2-3$ for each 535 experiment. 


\section{Figure 5: Loss of E-cadherin enhances anoikis resistance and increases cell survival. (A)} MCF7 and T47D cells were grown in 2D and ULA plates for 3-4 days and stained with Annexin $\mathrm{V}$ and propidium iodide to measure live and apoptotic cells, respectively. Live cell percentage in

2D for each cell line was used to normalize the live cell percentages in ULA plates. plated at 2,000 cells/well in full serum and low serum supplemented with 5nM IGF1 media and stained with $0.5 \%$ Crystal Violet after two to three weeks. Representative images are shown.

544 Plates were de-stained with 10\% acetic acid, absorbance quantified and graphed after normalization to WT cells of corresponding conditions. Statistical differences were evaluated using two-way ANOVA $\left({ }^{*} \mathrm{p}<0.05,{ }^{*} \mathrm{p}<0.01, * * * \mathrm{p}<0.001\right.$, representative experiment shown,

$547 \quad \mathrm{~N}=3$ (each with three biological replicates)). 
Figure 7: Loss of E-cadherin sensitizes cells to Fulvestrant and IGF1R/PI3K/Akt/MEK inhibitors in a context dependent manner. T47D parental and $C D H 1 \mathrm{KO}$ cells were seeded in 96-well 2D plates and treated with IGF1R inhibitors (OSI-906 or BMS-754807), PI3K inhibitor (Alpelisib) or Akt inhibitor (MK2206) for 6 days. Conditions in the panels as follows: (A) BMS754807; (B) OSI-906; (C) MK2206 and (D) Alpelisib. CellTiter Glo assay was used to assess

565 cell viability (relative luminescence) and data was normalized to vehicle treated control. $(\mathrm{E}, \mathrm{F})$

566 Cells were treated a combination of MEK inhibitor (U0126) and BMS-754807 for 6 days and

567 viability assessed as above. (G) Cells were treated a combination of Akt inhibitor (MK2206) and 568 Fulvestrant for 6 days and viability assessed as above. (H) Patient derived IDC and ILC

569 organoids were treated with Akt inhibitor (MK2206) for 12 days and viability assessed with

570 CellTiter Glo 3D (relative luminescence), and data was normalized to vehicle treated control.

571 IC50 values for viability were calculated by nonlinear regression and statistical differences

572 evaluated using sum-of-squares Global f-test $(\mathrm{p}<0.05$; representative experiment shown; $\mathrm{N}=3$

573 (each with six biological replicates)).

\section{Authors' Contributions}

576 Conception and design: A. Elangovan, S. Oesterreich, J.M. Atkinson, A.V. Lee

577 Development of methodology: A. Elangovan, J. Hooda, J. Chen, D.D. Brown, P.F. McAuliffe, S.

578 Oesterreich, J.M. Atkinson, A.V. Lee

579 Acquisition of data: A. Elangovan, J. Hooda, J. Chen, S. Oesterreich, J.M. Atkinson, A.V. Lee

580 Analysis and interpretation of data: A. Elangovan, J. Hooda, S. Puthanmadhomnarayanan, L.

581 Savariau, M.E. Yates, S. Oesterreich, J.M. Atkinson, A.V. Lee 
582 Writing, review, and/or revision of the manuscript: A. Elangovan, J. Hooda, S.

583 Puthanmadhomnarayanan, L. Savariau, M.E. Yates, J. Chen, D.D. Brown, P.F. McAuliffe, S.

584 Oesterreich, J.M. Atkinson, A.V. Lee

585 Administrative, technical, or material support: A. Elangovan, J. Hooda, L. Savariau, M.E. Yates,

586 J. Chen, D.D. Brown, P.F. McAuliffe, S. Oesterreich, J.M. Atkinson, A.V. Lee

587 Study supervision: A. Elangovan, S. Oesterreich, J.M. Atkinson, A.V. Lee

\section{Acknowledgements}

590

591

592

593

594

595

596

597

598

599

600

601

602

603

604

605

606

607
The authors would like to thank Osama Shah for data acquisition and bioinformatic input and the Institute for Precision Medicine, a partnership of the University of Pittsburgh and UPMC, for providing the breast cancer patient derived organoids used in these studies. This research was supported in part by the University of Pittsburgh Center for Research Computing through the resources provided.

\section{References}

1. Pestalozzi BC, Zahrieh D, Mallon E, Gusterson BA, Price KN, Gelber RD, et al. Distinct Clinical and Prognostic Features of Infiltrating Lobular Carcinoma of the Breast: Combined Results of 15 International Breast Cancer Study Group Clinical Trials. Journal of Clinical Oncology. 2008;26(18):3006-14.

2. Corben AD. Pathology of invasive breast disease. Surg Clin North Am. 2013;93(2):363-92.

3. Mamtani A, King TA. Lobular Breast Cancer: Different Disease, Different Algorithms? Surg Oncol Clin N Am. 2018;27(1):81-94.

4. Ciriello G, Gatza ML, Beck AH, Wilkerson MD, Rhie SK, Pastore A, et al. Comprehensive Molecular Portraits of Invasive Lobular Breast Cancer. Cell. 2015;163(2):506-19.

5. Du T, Zhu L, Levine KM, Tasdemir N, Lee AV, Vignali DAA, et al. Invasive lobular and ductal breast carcinoma differ in immune response, protein translation efficiency and metabolism. Sci Rep. 2018;8(1):7205. 
6. Rakha EA, Ellis IO. Lobular breast carcinoma and its variants. Semin Diagn Pathol. 2010;27(1):4961.

7. Chen Z, Yang J, Li S, Lv M, Shen Y, Wang B, et al. Invasive lobular carcinoma of the breast: A special histological type compared with invasive ductal carcinoma. PLoS One. 2017;12(9):e0182397. 8. Tasdemir N, Bossart EA, Li Z, Zhu L, Sikora MJ, Levine KM, et al. Comprehensive Phenotypic Characterization of Human Invasive Lobular Carcinoma Cell Lines in 2D and 3D Cultures. Cancer Res. 2018;78(21):6209-22.

9. Barroso-Sousa R, Metzger-Filho O. Differences between invasive lobular and invasive ductal carcinoma of the breast: results and therapeutic implications. Therapeutic Advances in Medical Oncology. 2016;8(4):261-6.

10. Vos CB, Cleton-Jansen AM, Berx G, de Leeuw WJ, ter Haar NT, van Roy F, et al. E-cadherin inactivation in lobular carcinoma in situ of the breast: an early event in tumorigenesis. Br J Cancer. 1997;76(9):1131-3.

11. Yoshiura K, Kanai Y, Ochiai A, Shimoyama Y, Sugimura T, Hirohashi S. Silencing of the E-cadherin invasion-suppressor gene by CpG methylation in human carcinomas. Proc Natl Acad Sci U S A. 1995;92(16):7416-9.

12. Desmedt C, Zoppoli G, Gundem G, Pruneri G, Larsimont D, Fornili M, et al. Genomic Characterization of Primary Invasive Lobular Breast Cancer. J Clin Oncol. 2016;34(16):1872-81. 13. Teo K, Gomez-Cuadrado L, Tenhagen M, Byron A, Ratze M, van Amersfoort M, et al. E-cadherin loss induces targetable autocrine activation of growth factor signalling in lobular breast cancer. Sci Rep. 2018;8(1):15454.

14. Becker MA, Ibrahim YH, Cui X, Lee AV, Yee D. The IGF Pathway Regulates ERa through a S6K1Dependent Mechanism in Breast Cancer Cells. Molecular Endocrinology. 2011;25(3):516-28.

15. Kim H-J, Litzenburger BC, Cui X, Delgado DA, Grabiner BC, Lin X, et al. Constitutively Active Type I Insulin-Like Growth Factor Receptor Causes Transformation and Xenograft Growth of Immortalized Mammary Epithelial Cells and Is Accompanied by an Epithelial-to-Mesenchymal Transition Mediated by NF-KB and Snail. Molecular and Cellular Biology. 2007;27(8):3165-75.

16. Yee D, Lee AV. Journal of Mammary Gland Biology and Neoplasia. 2000;5(1):107-15.

17. Hawsawi Y, El-Gendy R, Twelves C, Speirs V, Beattie J. Insulin-like growth factor - Oestradiol crosstalk and mammary gland tumourigenesis. Biochimica et Biophysica Acta (BBA) - Reviews on Cancer. 2013;1836(2):345-53.

18. Baserga R, Peruzzi F, Reiss K. The IGF-1 receptor in cancer biology. Int J Cancer. 2003;107(6):8737.

19. Litzenburger BC, Kim HJ, Kuiatse I, Carboni JM, Attar RM, Gottardis MM, et al. BMS-536924 reverses IGF-IR-induced transformation of mammary epithelial cells and causes growth inhibition and polarization of MCF7 cells. Clin Cancer Res. 2009;15(1):226-37.

20. lams WT, Lovly CM. Molecular Pathways: Clinical Applications and Future Direction of Insulinlike Growth Factor-1 Receptor Pathway Blockade. Clinical Cancer Research. 2015;21(19):4270-7. 21. Morgillo F, De Vita F, Antoniol G, Orditura M, Auriemma PP, Diadema MR, et al. Serum insulinlike growth factor 1 correlates with the risk of nodal metastasis in endocrine-positive breast cancer. Curr Oncol. 2013;20(4):e283-8.

22. Crudden C, Girnita A, Girnita L. Targeting the IGF-1R: The Tale of the Tortoise and the Hare. Front Endocrinol (Lausanne). 2015;6:64.

23. Boone DN, Lee AV. Targeting the Insulin-like Growth Factor Receptor: Developing Biomarkers from Gene Expression Profiling. Critical Reviews ${ }^{\mathrm{TM}}$ in Oncogenesis. 2012;17(2):161-73.

24. Singh P, Alex JM, Bast F. Insulin receptor (IR) and insulin-like growth factor receptor 1 (IGF-1R) signaling systems: novel treatment strategies for cancer. Med Oncol. 2014;31(1):805. 
25. Ekyalongo RC, Yee D. Revisiting the IGF-1R as a breast cancer target. npj Precision Oncology. 2017;1(1).

26. Yang $Y$, Yee D. Targeting insulin and insulin-like growth factor signaling in breast cancer. J Mammary Gland Biol Neoplasia. 2012;17(3-4):251-61.

27. Pappo AS, Patel SR, Crowley J, Reinke DK, Kuenkele K-P, Chawla SP, et al. R1507, a Monoclonal Antibody to the Insulin-Like Growth Factor 1 Receptor, in Patients With Recurrent or Refractory Ewing Sarcoma Family of Tumors: Results of a Phase II Sarcoma Alliance for Research Through Collaboration Study. Journal of Clinical Oncology. 2011;29(34):4541-7.

28. Pappo AS, Vassal G, Crowley JJ, Bolejack V, Hogendoorn PCW, Chugh R, et al. A phase 2 trial of R1507, a monoclonal antibody to the insulin-like growth factor-1 receptor (IGF-1R), in patients with recurrent or refractory rhabdomyosarcoma, osteosarcoma, synovial sarcoma, and other soft tissue sarcomas: Results of a Sarcoma Alliance. Cancer. 2014;120(16):2448-56.

29. Langer CJ, Novello S, Park K, Krzakowski M, Karp DD, Mok T, et al. Randomized, phase III trial of first-line figitumumab in combination with paclitaxel and carboplatin versus paclitaxel and carboplatin alone in patients with advanced non-small-cell lung cancer. J Clin Oncol. 2014;32(19):2059-66.

30. Qian X, Karpova T, Sheppard AM, McNally J, Lowy DR. E-cadherin-mediated adhesion inhibits ligand-dependent activation of diverse receptor tyrosine kinases. EMBO J. 2004;23(8):1739-48.

31. Tasdemir N, Ding K, Savariau L, Levine KM, Du T, Elangovan A, et al. Proteomic and transcriptomic profiling identifies mediators of anchorage-independent growth and roles of inhibitor of differentiation proteins in invasive lobular carcinoma. Sci Rep. 2020;10(1):11487.

32. Erdem C, Nagle AM, Casa AJ, Litzenburger BC, Wang YF, Taylor DL, et al. Proteomic Screening and Lasso Regression Reveal Differential Signaling in Insulin and Insulin-like Growth Factor I (IGF1) Pathways. Mol Cell Proteomics. 2016;15(9):3045-57.

33. Nagle AM, Levine KM, Tasdemir N, Scott JA, Burlbaugh K, Kehm J, et al. Loss of E-cadherin Enhances IGF1-IGF1R Pathway Activation and Sensitizes Breast Cancers to Anti-IGF1R/InsR Inhibitors. Clinical Cancer Research. 2018;24(20):5165-77.

34. Chen F, Ding K, Priedigkeit N, Elangovan A, Levine KM, Carleton N, et al. Single-Cell Transcriptomic Heterogeneity in Invasive Ductal and Lobular Breast Cancer Cells. Cancer Res. 2021;81(2):268-81.

35. lanevski A, He L, Aittokallio T, Tang J. SynergyFinder: a web application for analyzing drug combination dose-response matrix data. Bioinformatics. 2020;36(8):2645.

36. Brueffer C, Gladchuk S, Winter C, Vallon-Christersson J, Hegardt C, Hakkinen J, et al. The mutational landscape of the SCAN-B real-world primary breast cancer transcriptome. EMBO Mol Med. 2020;12(10):e12118.

37. Yoshihara K, Shahmoradgoli M, Martinez E, Vegesna R, Kim H, Torres-Garcia W, et al. Inferring tumour purity and stromal and immune cell admixture from expression data. Nat Commun. 2013;4:2612.

38. Hanzelmann S, Castelo R, Guinney J. GSVA: gene set variation analysis for microarray and RNAseq data. BMC Bioinformatics. 2013;14:7.

39. Subramanian A, Tamayo P, Mootha VK, Mukherjee S, Ebert BL, Gillette MA, et al. Gene set enrichment analysis: a knowledge-based approach for interpreting genome-wide expression profiles. Proc Natl Acad Sci U S A. 2005;102(43):15545-50.

40. Sachs N, de Ligt J, Kopper O, Gogola E, Bounova G, Weeber F, et al. A Living Biobank of Breast Cancer Organoids Captures Disease Heterogeneity. Cell. 2018;172(1-2):373-86 e10.

41. Ikediobi ON, Davies H, Bignell G, Edkins S, Stevens C, O'Meara S, et al. Mutation analysis of 24 known cancer genes in the NCl-60 cell line set. Mol Cancer Ther. 2006;5(11):2606-12. 
42. Marcotte R, Sayad A, Brown KR, Sanchez-Garcia F, Reimand J, Haider M, et al. Functional Genomic Landscape of Human Breast Cancer Drivers, Vulnerabilities, and Resistance. Cell. 2016;164(12):293-309.

43. Hollestelle A, Nagel JHA, Smid M, Lam S, Elstrodt F, Wasielewski M, et al. Distinct gene mutation profiles among luminal-type and basal-type breast cancer cell lines. Breast Cancer Research and Treatment. 2010;121(1):53-64.

44. AndI CD, Rustgi AK. No one-way street: Cross-talk between E-cadherin and receptor tyrosine kinase (RTK) signaling-A mechanism to regulate RTK activity. Cancer Biology \& Therapy. 2005;4(1):35-8. 45. De-Freitas-Junior JCM, Carvalho S, Dias AM, Oliveira P, Cabral J, Seruca R, et al. Insulin/IGF-I Signaling Pathways Enhances Tumor Cell Invasion through Bisecting GIcNAc N-glycans Modulation. An Interplay with E-Cadherin. PLoS ONE. 2013;8(11):e81579.

46. Curto M, Cole BK, Lallemand D, Liu CH, McClatchey Al. Contact-dependent inhibition of EGFR signaling by Nf2/Merlin. J Cell Biol. 2007;177(5):893-903.

47. Lombaerts M, Van Wezel T, Philippo K, Dierssen JWF, Zimmerman RME, Oosting J, et al. Ecadherin transcriptional downregulation by promoter methylation but not mutation is related to epithelial-to-mesenchymal transition in breast cancer cell lines. British Journal of Cancer. 2006;94(5):661-71.

48. Kenny PA, Lee GY, Myers CA, Neve RM, Semeiks JR, Spellman PT, et al. The morphologies of breast cancer cell lines in three-dimensional assays correlate with their profiles of gene expression. Molecular Oncology. 2007;1(1):84-96.

49. Sarrió D, Pérez-Mies B, Hardisson D, Moreno-Bueno G, Suárez A, Cano A, et al. Cytoplasmic localization of p120ctn and E-cadherin loss characterize lobular breast carcinoma from preinvasive to metastatic lesions. Oncogene. 2004;23(19):3272-83.

50. Garcia-Recio S, Thennavan A, East MP, Parker JS, Cejalvo JM, Garay JP, et al. FGFR4 regulates tumor subtype differentiation in luminal breast cancer and metastatic disease. J Clin Invest. 2020;130(9):4871-87.

51. Levine KM, Priedigkeit N, Basudan A, Tasdemir N, Sikora MJ, Sokol ES, et al. FGFR4 overexpression and hotspot mutations in metastatic ER+ breast cancer are enriched in the lobular subtype. NPJ Breast Cancer. 2019;5:19.

52. Schackmann RCJ, Van Amersfoort M, Haarhuis JHI, Vlug EJ, Halim VA, Roodhart JML, et al. Cytosolic p120-catenin regulates growth of metastatic lobular carcinoma through Rock1-mediated anoikis resistance. Journal of Clinical Investigation. 2011;121(8):3176-88.

53. Onder TT, Gupta PB, Mani SA, Yang J, Lander ES, Weinberg RA. Loss of E-Cadherin Promotes Metastasis via Multiple Downstream Transcriptional Pathways. Cancer Research. 2008;68(10):3645-54. 54. Valastyan S, Weinberg RA. Tumor metastasis: molecular insights and evolving paradigms. Cell. 2011;147(2):275-92.

55. Petrova YI, Schecterson L, Gumbiner BM. Roles for E-cadherin cell surface regulation in cancer. Molecular Biology of the Cell. 2016;27(21):3233-44.

56. Padmanaban V, Krol I, Suhail Y, Szczerba BM, Aceto N, Bader JS, et al. E-cadherin is required for metastasis in multiple models of breast cancer. Nature. 2019;573(7774):439-44.

57. Michaut M, Chin SF, Majewski I, Severson TM, Bismeijer T, de Koning L, et al. Integration of genomic, transcriptomic and proteomic data identifies two biologically distinct subtypes of invasive lobular breast cancer. Sci Rep. 2016;6:18517.

58. Guvakova MA, Surmacz E. Overexpressed IGF-I receptors reduce estrogen growth requirements, enhance survival, and promote E-cadherin-mediated cell-cell adhesion in human breast cancer cells. Exp Cell Res. 1997;231(1):149-62. 
59. Canonici A, Steelant W, Rigot V, Khomitch-Baud A, Boutaghou-Cherid H, Bruyneel E, et al. Insulin-like growth factor-I receptor, E-cadherin and alpha $v$ integrin form a dynamic complex under the control of alpha-catenin. Int J Cancer. 2008;122(3):572-82.

750 60. Bae G-Y, Choi S-J, Lee J-S, Jo J, Lee J, Kim J, et al. Loss of E-cadherin activates EGFR-MEK/ERK 751 signaling, which promotes invasion via the ZEB1/MMP2 axis in non-small cell lung cancer. Oncotarget. 752 2013;4(12):2512-22.

753 61. St John MA. Inflammatory mediators drive metastasis and drug resistance in head and neck 754 squamous cell carcinoma. Laryngoscope. 2015;125 Suppl 3:S1-11.

755 62. Jeanes A, Gottardi CJ, Yap AS. Cadherins and cancer: how does cadherin dysfunction promote 756 tumor progression? Oncogene. 2008;27(55):6920-9.

757 63. Fedor-Chaiken M, Hein PW, Stewart JC, Brackenbury R, Kinch MS. E-Cadherin Binding Modulates 758 EGF Receptor Activation. Cell Communication \& Adhesion. 2003;10(2):105-18.

759 64. Petrova YI, Schecterson L, Gumbiner BM. Roles for E-cadherin cell surface regulation in cancer. 760 Mol Biol Cell. 2016;27(21):3233-44.

761 65. Bajrami I, Marlow R, van de Ven M, Brough R, Pemberton HN, Frankum J, et al. E-Cadherin/ROS1 762 Inhibitor Synthetic Lethality in Breast Cancer. Cancer Discov. 2018;8(4):498-515.

763 66. Zardavas D, Phillips WA, Loi S. PIK3CA mutations in breast cancer: reconciling findings from 764 preclinical and clinical data. Breast Cancer Research. 2014;16(1):201.

765 67. Wang $Q$, Zhang Y, Zhu J, Zheng H, Chen S, Chen L, et al. IGF-1R inhibition induces MEK 766 phosphorylation to promote survival in colon carcinomas. Signal Transduction and Targeted Therapy. 767 2020;5(1).

768 68. Chandarlapaty S, Sawai A, Scaltriti M, Rodrik-Outmezguine V, Grbovic-Huezo O, Serra V, et al. 769 AKT Inhibition Relieves Feedback Suppression of Receptor Tyrosine Kinase Expression and Activity. 770 Cancer Cell. 2011;19(1):58-71. 
A

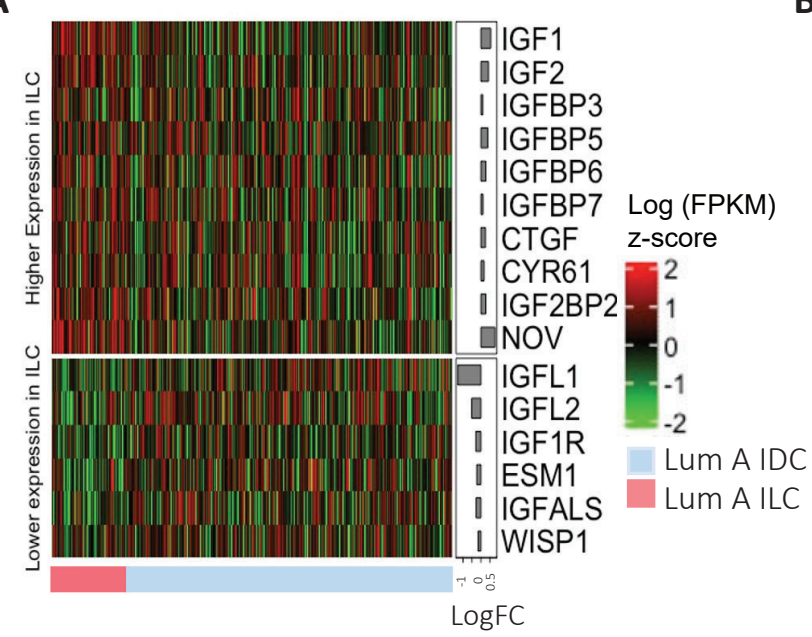

C Reactome PI3K AKT in cancer

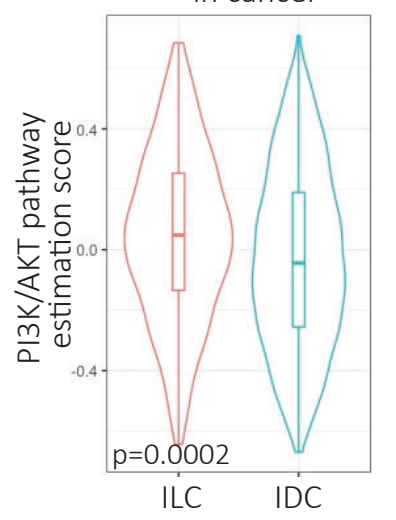

Reactome PI3K AKT

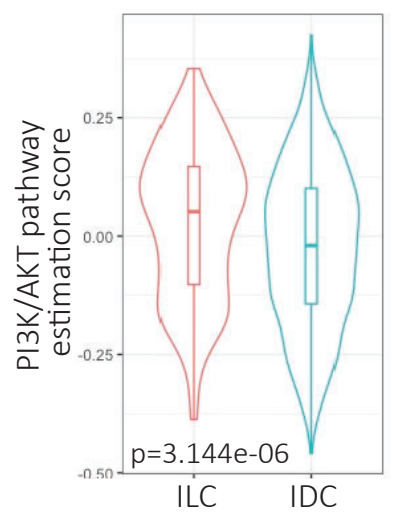

B

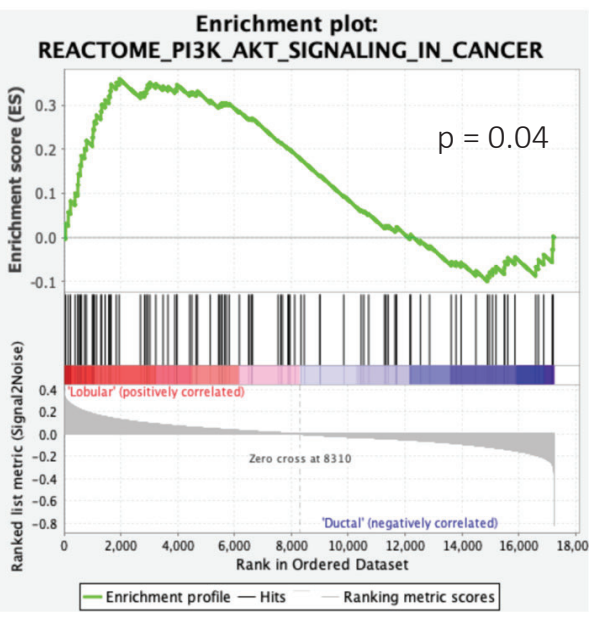

E

D Pacher targets of

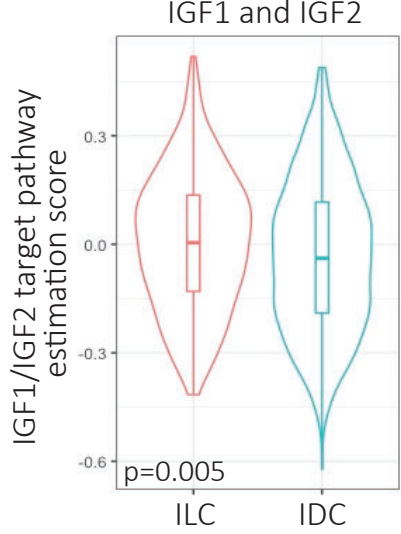

IGF1

IGF2

\begin{tabular}{|c|c|c|c|c|c|c|c|c|c|}
\hline \multicolumn{3}{|c|}{ IDC } & \multicolumn{2}{|c|}{ ILC } & \multicolumn{3}{|c|}{ IDC } & \multicolumn{2}{|c|}{ ILC } \\
\hline $\begin{array}{l}\text { ÜU } \\
\stackrel{U}{\Sigma}\end{array}$ & $\stackrel{\stackrel{P}{\&}}{\stackrel{f}{⺊}}$ & $\begin{array}{l}\stackrel{-}{n} \\
\stackrel{n}{N} \\
\frac{r}{N}\end{array}$ & $\sum_{\sum}^{\stackrel{J}{G}}$ & 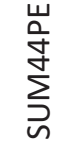 & $\stackrel{\vec{U}}{\Sigma}$ & $\underset{\leftarrow}{\stackrel{P}{f}}$ & $\begin{array}{l}\stackrel{+}{N} \\
\stackrel{\mathfrak{n}}{N}\end{array}$ & $\sum_{\sum}^{\stackrel{J}{F}}$ & 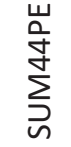 \\
\hline
\end{tabular}

Ligand (nM) $0 \begin{array}{llllllllllllllllllll} & 5 & 0 & 5 & 0 & 5 & 0 & 5 & 0 & 5 & 0 & 5 & 0 & 5 & 0 & 5 & 0 & 5 & 0 & 5\end{array}$

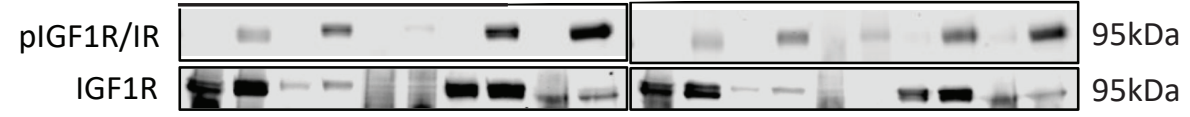

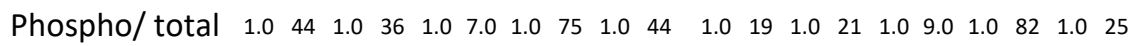

pAkt (S473) - - - - - - - - $60 \mathrm{kDa}$

Akt

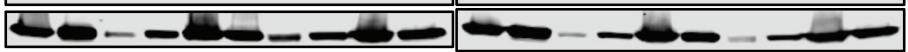

$60 \mathrm{kDa}$

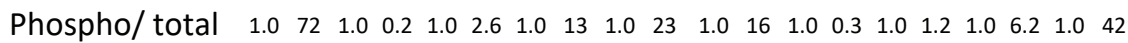

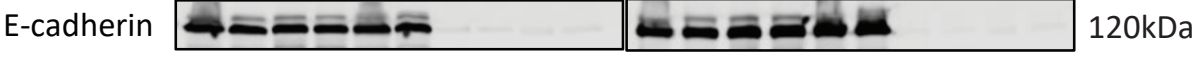

$\beta$-actin

Figure 1: IGF pathway activity is enhanced in ILC patient samples and cell lines in vitro. (A) Gene expression analysis of growth factor signaling related genes comparing Luminal A IDC and ILC with significantly different expression values displayed. (B) GSEA analysis for PI3K/Akt showing significantly higher pathway activation in ILC samples obtained from the SCANB dataset. (C, D) GSVA analysis of Luminal A IDC and ILC samples using respective signature sets from MSigDB with significantly higher pathway activation in ILC. (E) IDC cells (MCF7, T47D, ZR75.1) and ILC cells (MDA-MB-134-VI, SUM44PE) were stimulated with doses of IGF1 or IGF2 (0-5nM) for 15 minutes following an overnight serum starvation. IGF1R/IR and Akt signaling was assessed by Western blotting. Phosphorylation levels of IGF1R (phospho-IGF-I Receptor $\beta$ (Tyr1135/1136)/Insulin Receptor $\beta$ (Tyr1150/1151)) and Akt (S473) were quantified on the LiCOr Odyssey CLx Imaging system and normalized to corresponding total protein levels and loading controls. Ligand treated sample values were further normalized to respective cell line vehicle treated samples. Representative experiment shown for all, $\mathrm{N}=2-3$ for each experiment. 
A

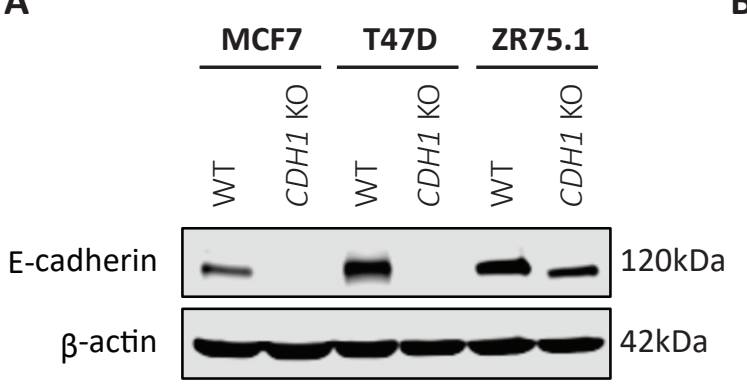

C

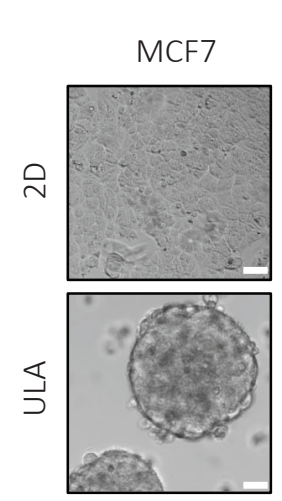

D
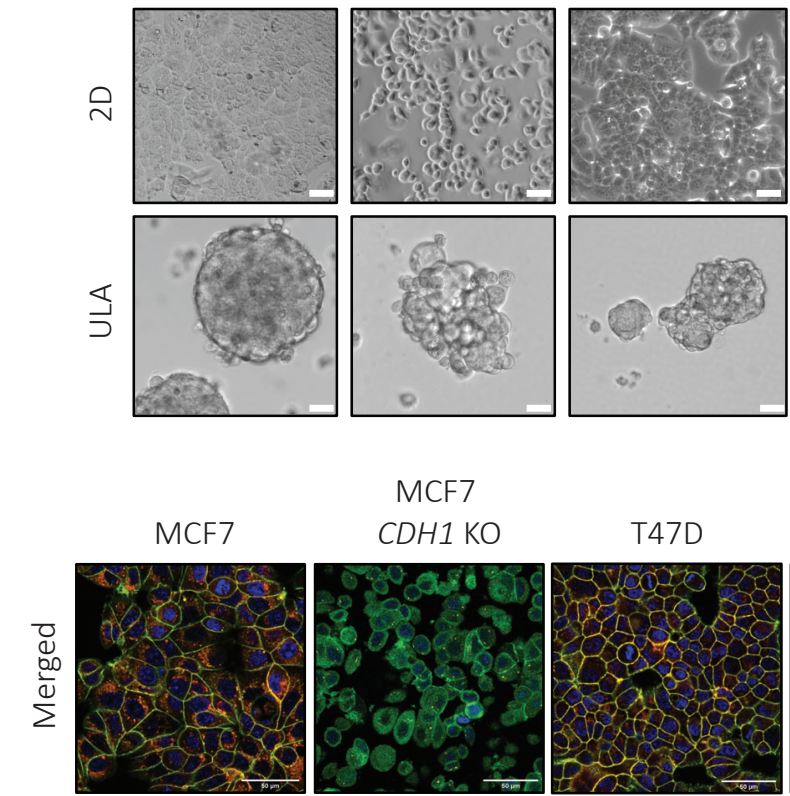

MCF7

CDH1 KO
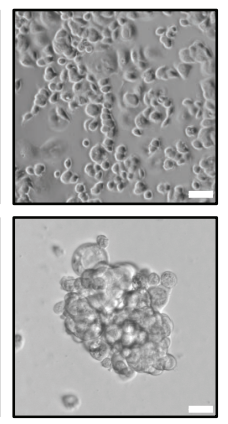

MCF7

$\mathrm{CDH} 1 \mathrm{KO}$
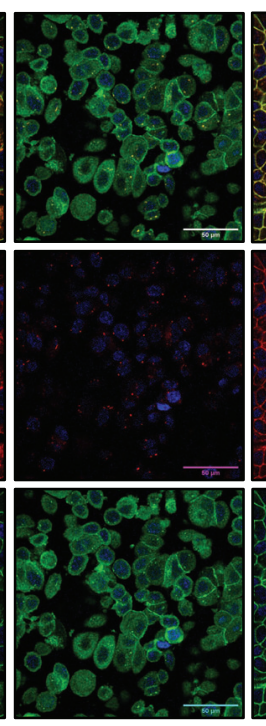

B

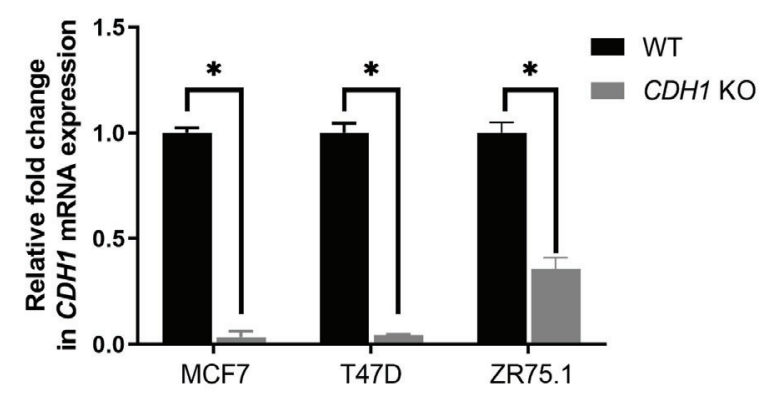

T47D

CDH1 KO
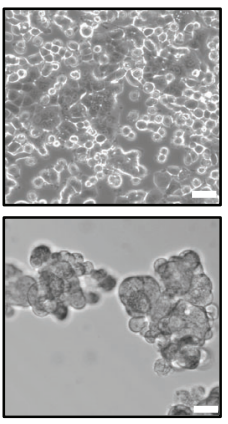

T47D

$\mathrm{CDH} 1 \mathrm{KO}$
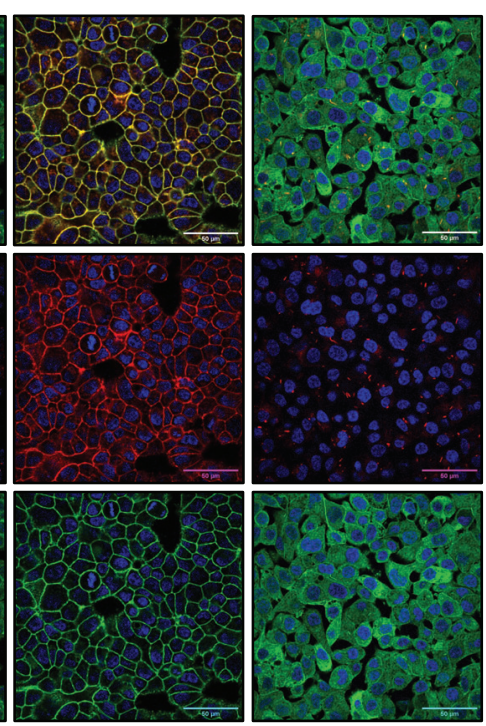

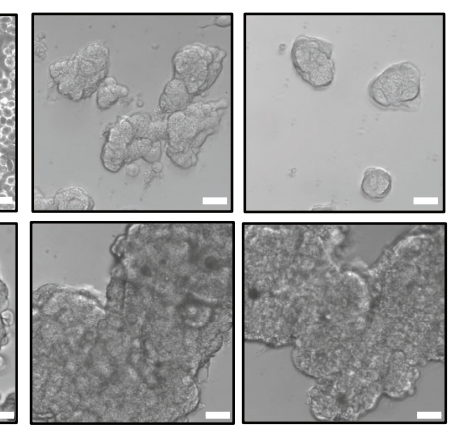

ZR75.1

ZR75.1

$\mathrm{CDH} 1 \mathrm{KO}$

ZR75.1

ZR75.1

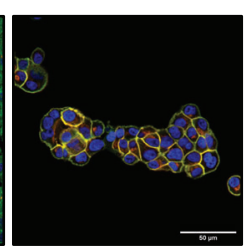

$\mathrm{CDH} 1 \mathrm{KO}$
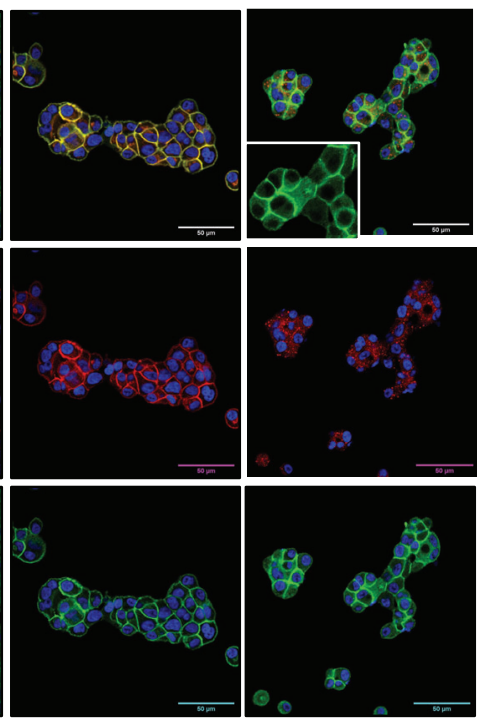

Figure 2: $\mathrm{CDH} 1$ knockout cells as a model to study the role of E-cadherin in regulating the IGF pathway (A) Western blotting and (B) qRT-PCR confirms reduction of E-cadherin in CDH1 KO MCF7, T47D and ZR75.1 CRISPR cell lines compared to wildtype (WT) parental cells. Statistical differences evaluated using paired t-test $\left({ }^{*} p<0.05\right)$. Representative experiment shown, $\mathrm{N}=2$ (each with two biological and 3 technical replicates). (C) Representative brightfield images (10X magnification) of WT and CDH1 KO cell line models plated in 2D

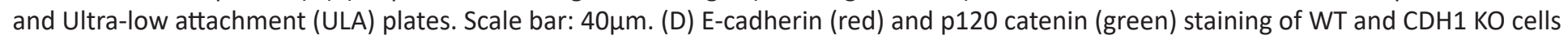
confirms CDH1 loss and p120 re-localization in CDH1 KO cell models by confocal microscopy (60X objective). Inset in the ZR75.1 CDH1 KO

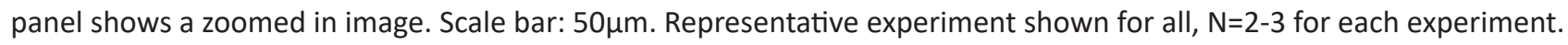


A

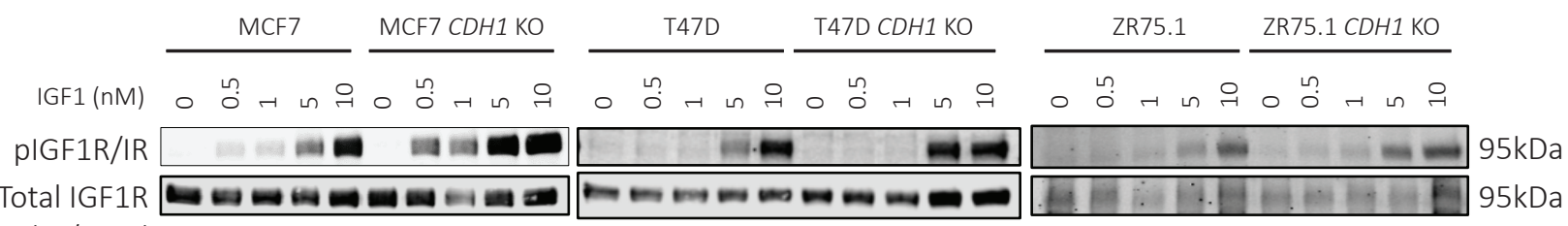

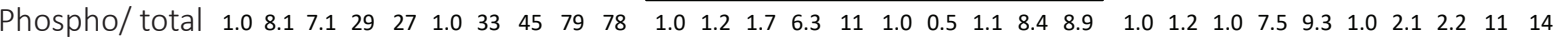

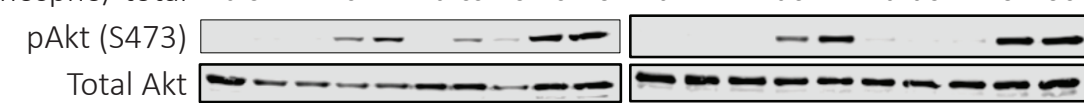

Phospho/total $1.017 \quad 12 \quad 50 \quad 64 \begin{array}{lllllllllllllllll}1.0 & 15 & 13 & 54 & 50 & 1.0 & 1.0 & 1.2 & 6.1 & 10 & 1.0 & 1.5 & 1.4 & 10 & 11\end{array}$
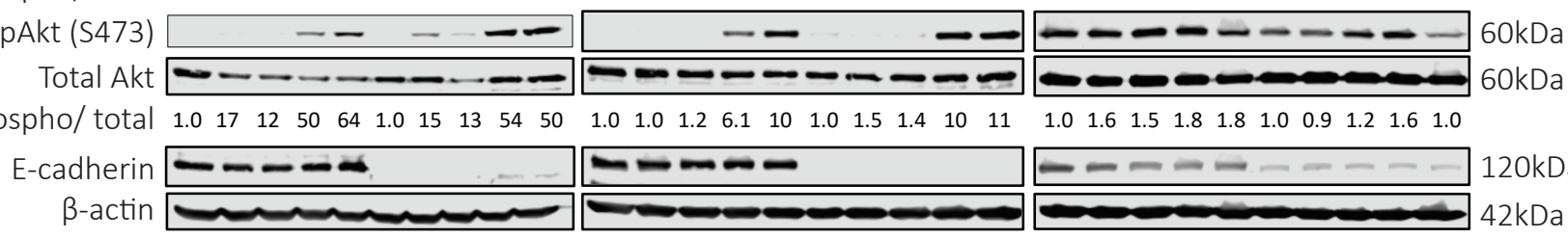

$\begin{array}{lllllllllll}1.0 & 1.6 & 1.5 & 1.8 & 1.8 & 1.0 & 0.9 & 1.2 & 1.6 & 1.0\end{array}$

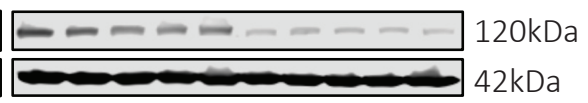

B

\begin{tabular}{|c|c|c|c|c|c|}
\hline & MCF7 & & T47D & & ZR75.1 \\
\hline MCF7 & $\mathrm{CDH} 1 \mathrm{KO}$ & T47D & $\mathrm{CDH} 1 \mathrm{KO}$ & ZR75.1 & $\mathrm{CDH} 1 \mathrm{KO}$ \\
\hline
\end{tabular}

C

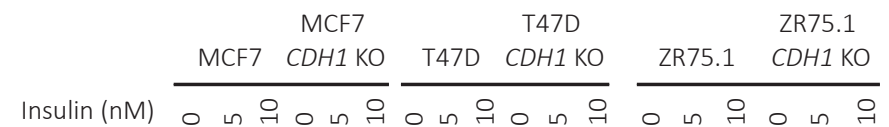

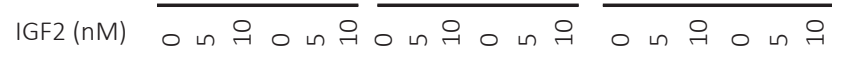

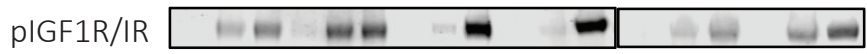

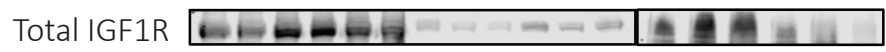

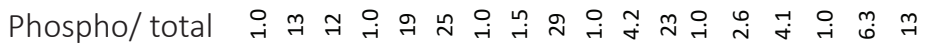

pAkt (S473) - - - -

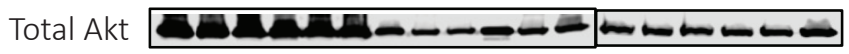

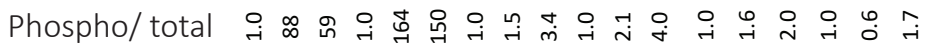

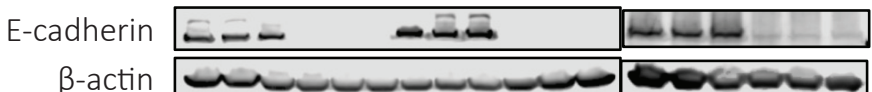

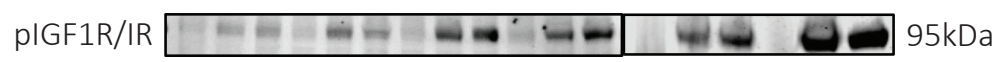

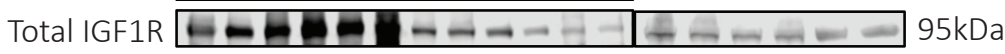

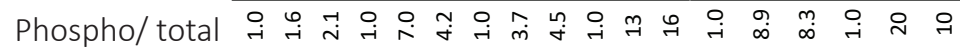

pAkt (S473) - - -

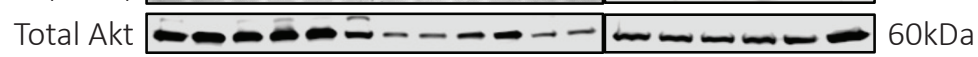

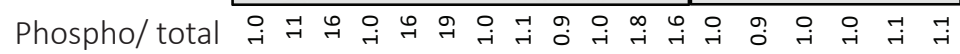

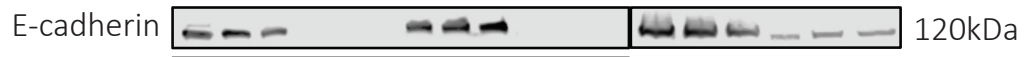

$\beta$-actin $42 \mathrm{kDa}$

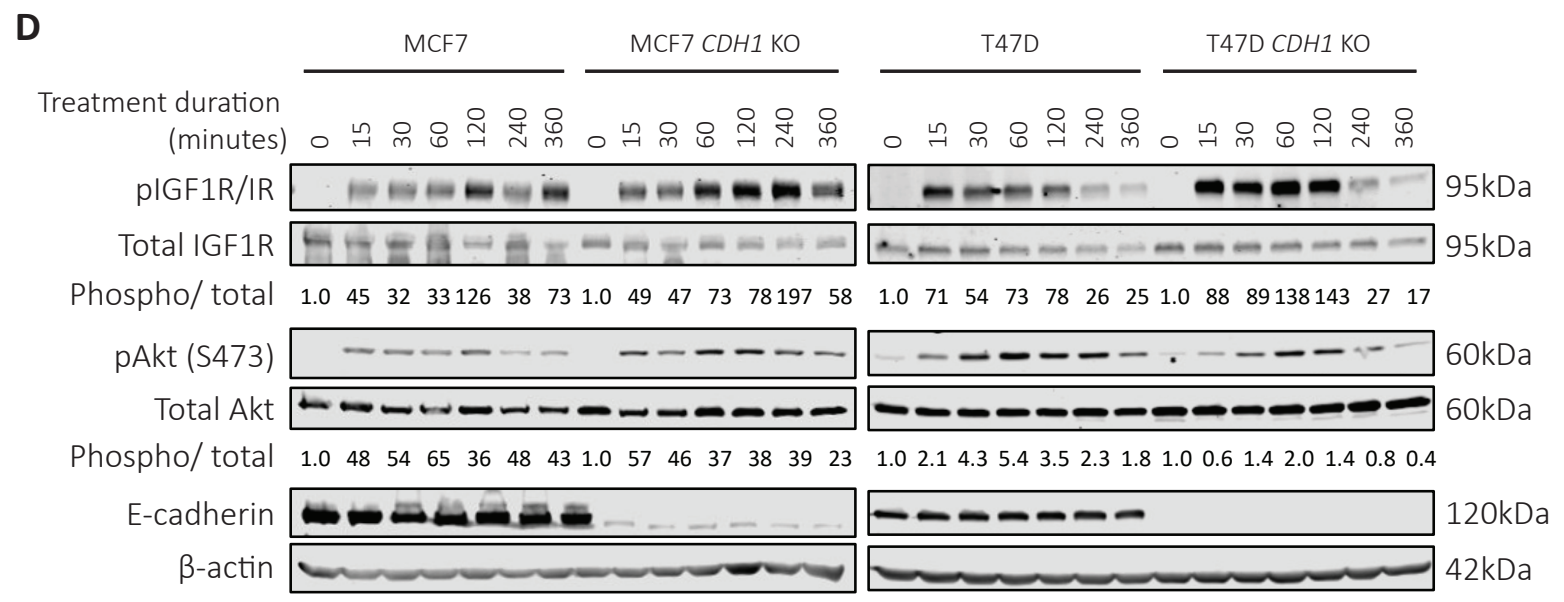

Figure 3: Loss of E-cadherin sensitizes cells to IGF signaling pathway activation. Cells were serum starved overnight and stimulated with (A) IGF1, (B) IGF2 or (C) Insulin (0-10nM) for 15 minutes. Cells were harvested for Western blot to assess IGF1R/IR and Akt signaling. For quantification, phosphorylated protein levels were normalized to corresponding total protein levels and loading controls. Ligand treated sample values were further normalized to respective cell line vehicle treated samples. (D) Cells were treated with $10 \mathrm{nM}$ IGF1 for a time course from 0-6 hours to assess the duration of signaling activity between WT and CDH1 KO cells. Representative experiment shown for all, $\mathrm{N}=2-3$ for each experiment. 
A

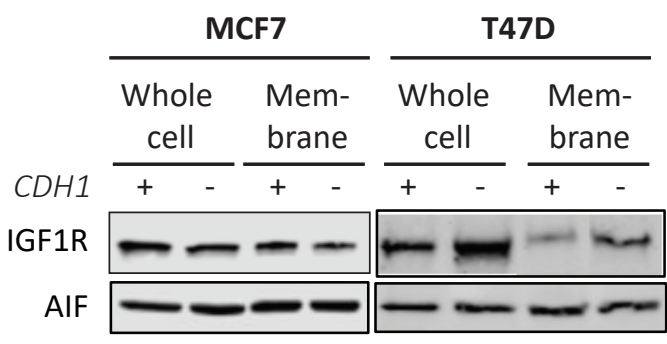

IGF1R/AIF $\quad \begin{array}{llllllll}1.00 & 0.88 & 1.00 & 1.12 & 1.00 & 1.59 & 1.00 & 1.28\end{array}$

E-cadherin

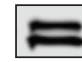

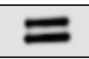

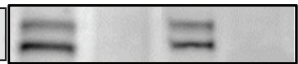

ZR75.1

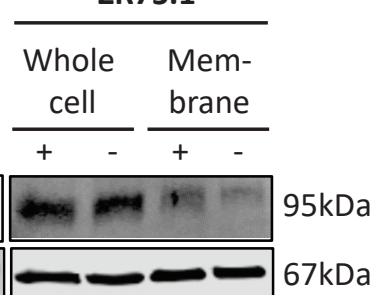

$\begin{array}{llll}1.00 & 1.03 & 1.00 & 0.88\end{array}$
C

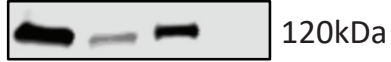

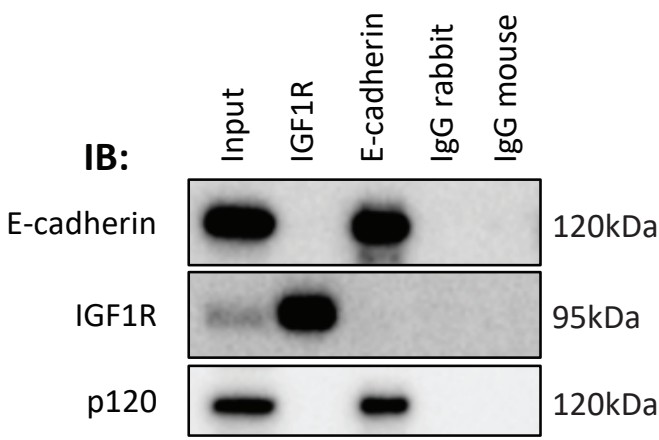

B MCF7

T47D

ZR75.1

\begin{tabular}{|c|c|c|c|c|c|c|c|c|c|c|c|c|c|c|c|c|c|c|}
\hline \multirow[b]{2}{*}{ iotinylated IGF1 (nM) } & \multicolumn{3}{|c|}{ MCF7 } & \multicolumn{3}{|c|}{$\mathrm{CDH} 1 \mathrm{KO}$} & \multicolumn{3}{|c|}{ T47D } & \multicolumn{3}{|c|}{$\mathrm{CDH} 1 \mathrm{KO}$} & \multicolumn{3}{|c|}{ ZR75.1 } & \multicolumn{3}{|c|}{$\mathrm{CDH1} \mathrm{KO}$} \\
\hline & 0 & 5 & 10 & 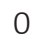 & 5 & 10 & 0 & 5 & 10 & 0 & 5 & 10 & 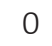 & 5 & 10 & 0 & 5 & 10 \\
\hline -Biotin & & & $=$ & & $=$ & 7 & $\theta$ & tren & $m$ & & $=$ & 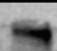 & & & ina & & & \\
\hline$\alpha$-Biotin/ $\beta$-actin & 1.0 & 1.2 & 3.0 & 1.0 & 2.5 & 4.1 & 1.0 & 1.8 & 4.4 & 1.0 & 7.2 & 8.3 & 1.0 & .1 & 1.6 & 1.0 & 1.5 & \\
\hline
\end{tabular}

IGF1R (95kDa)

E-cadherin (120kDa)

$\beta$-actin (42kDa)

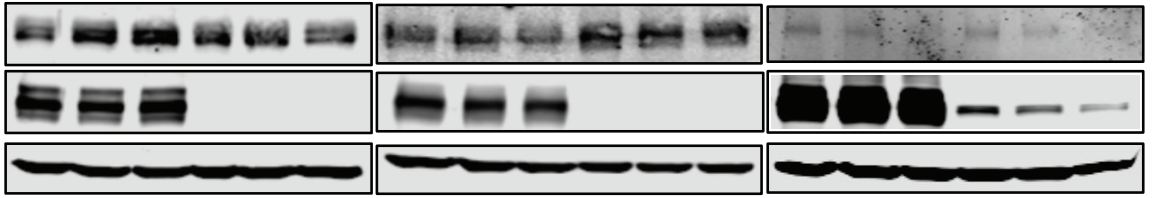

D

MCF7

MCF7 CDH1 KO

T47D

T47D CDH1 KO
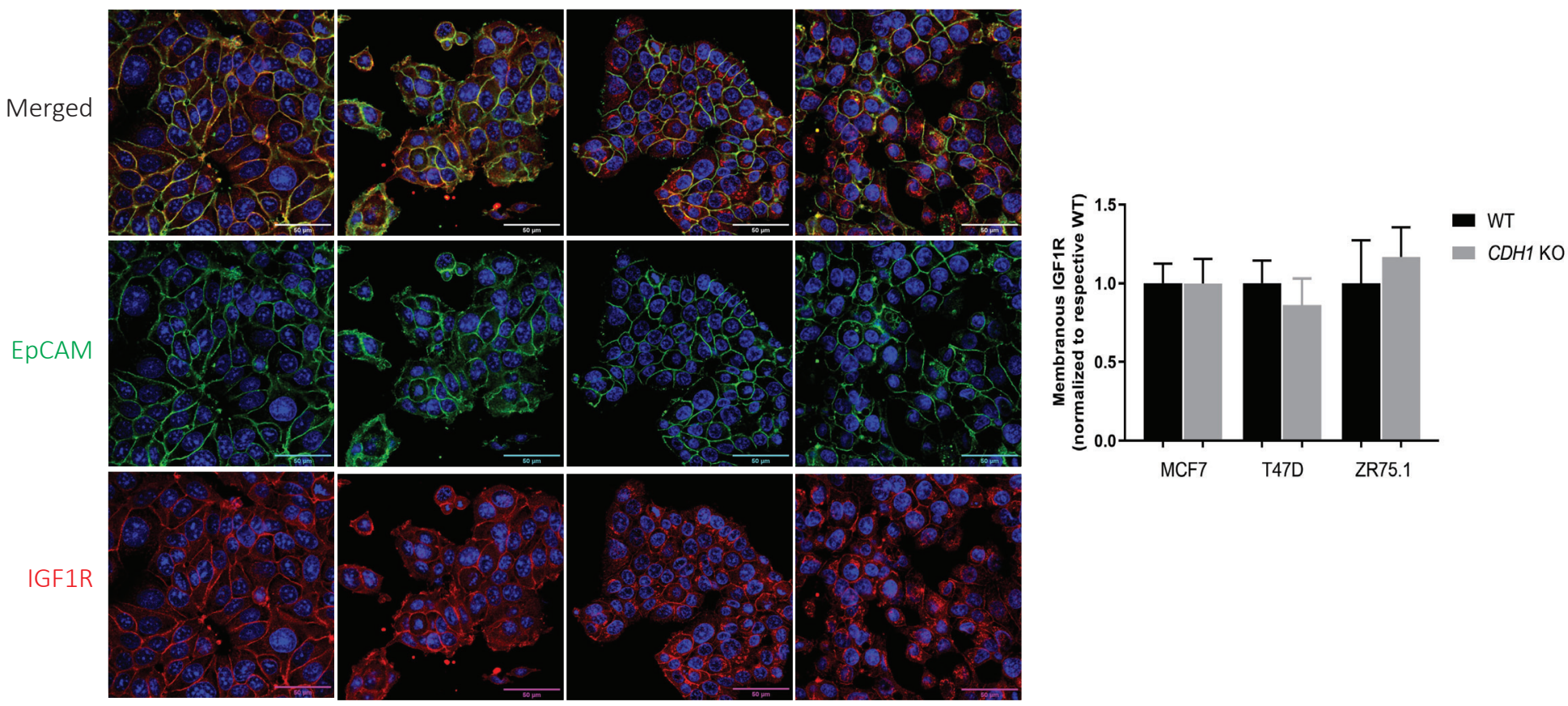

Figure 4: Loss of E-cadherin increases IGF1R availability on the membrane to allow ligand binding. (A) Cell fractionation assay performed on MCF7, T47D and ZR75.1 WT and CDH1 KO cells to assess whole cell and membrane IGF1R expression levels. IGF1R bands were quantified and normalized to membrane control, AIF1. (B) Cells were stimulated with biotinylated IGF1 (0-10nM) for 10 minutes and crosslinked to assess ligand-receptor complex levels between WT and CDH1 KO cells. (C) Immunoprecipitation of IGF1R and E-cadherin in T47D cells was assessed for a co-IP of other proteins, with $p-120$ catenin assessed as a known interactor of E-cadherin. (D) Cell lines were

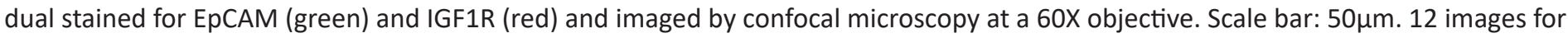
each cell line were quantified and graphed. Representative experiment shown for all, $\mathrm{N}=2-3$ for each experiment. 
A
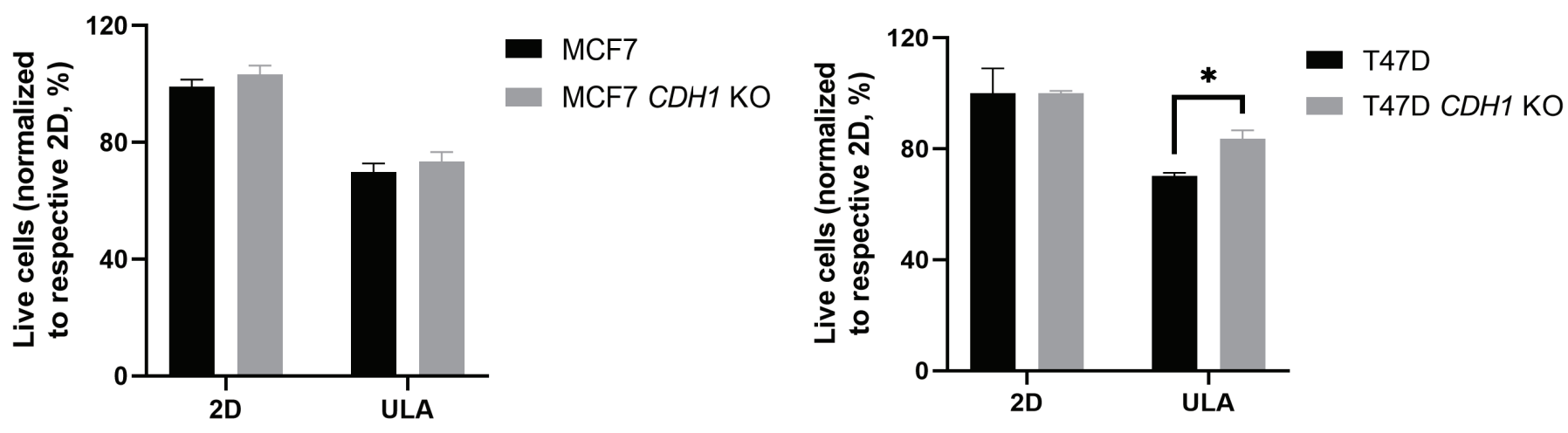

B

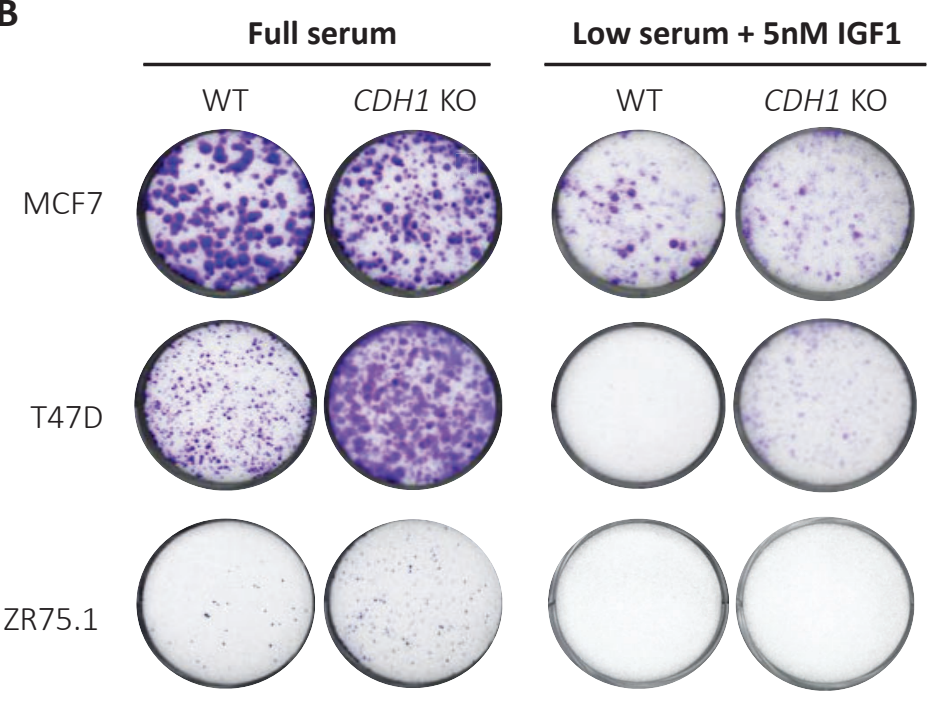

Full serum

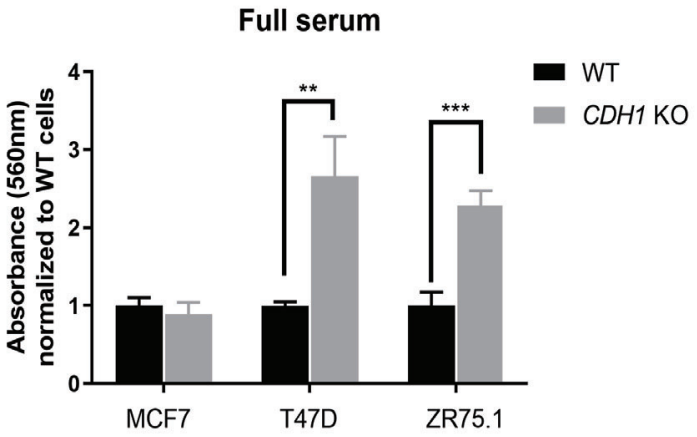

Low serum + 5nM IGF1

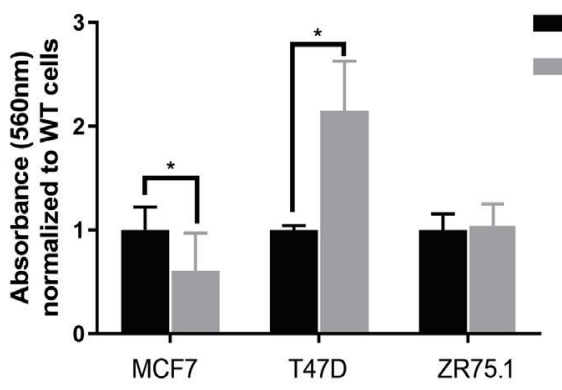

Figure 5: Loss of E-cadherin enhances anoikis resistance and increases cell survival. (A) MCF7 and T47D cells were grown in 2D and ULA plates for 3-4 days and stained with Annexin $V$ and propidium iodide to measure live and apoptotic cells, respectively. Live cell percentage in $2 \mathrm{D}$ for each cell line was used to normalize the live cell percentages in ULA plates. Representative experiment shown; $N=3$ (each with three biological replicates). (B) Cells were plated at 2,000 cells/well in full serum and low serum supplemented with 5nM IGF1 media and stained with $0.5 \%$ Crystal Violet after two to three weeks. Representative images are shown. Plates were de-stained with $10 \%$ acetic acid, absorbance quantified and graphed after normalization to WT cells of corresponding conditions. Statistical differences were evaluated using two-way ANOVA $\left({ }^{*} \mathrm{p}<0.05,{ }^{* *} \mathrm{p}<0.01,{ }^{* *} \mathrm{p}<0.001\right.$, representative experiment shown, $\mathrm{N}=3$ (each with three biological replicates)). 
A

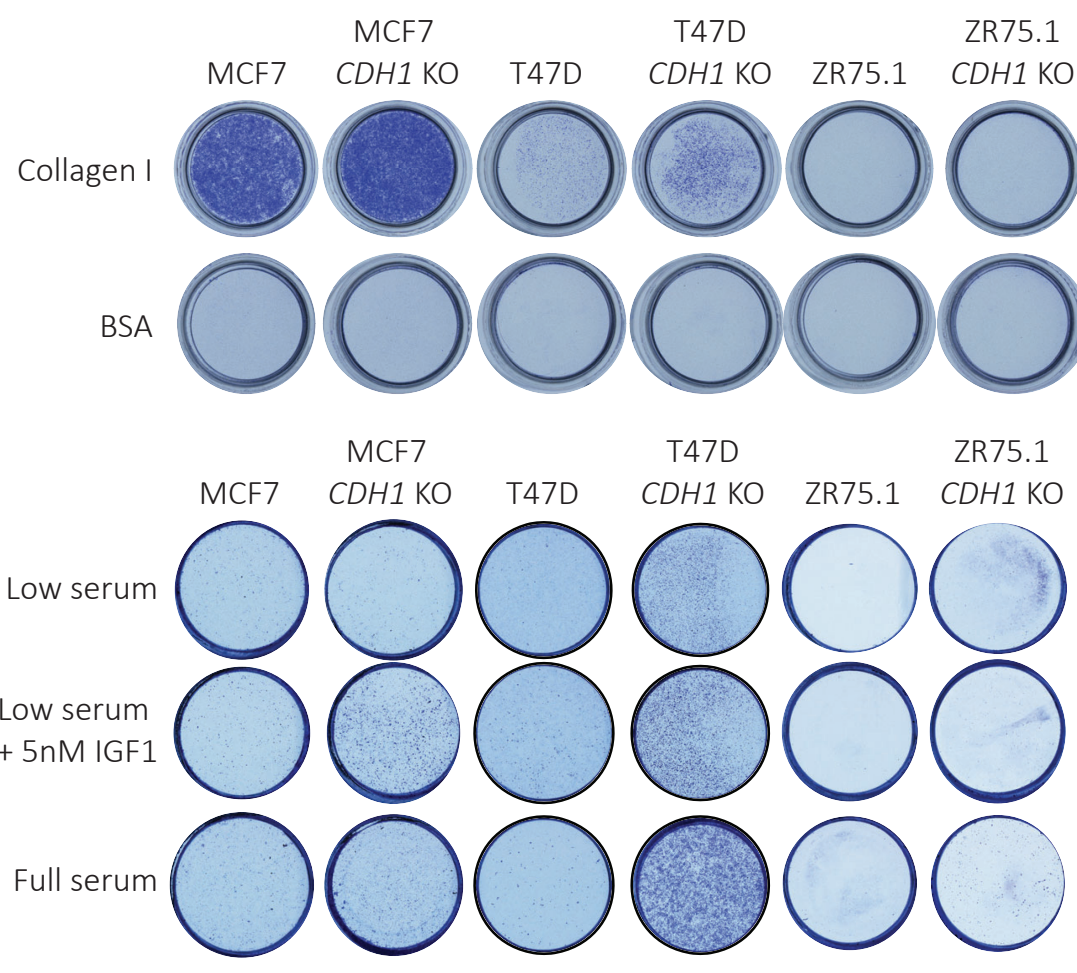

C
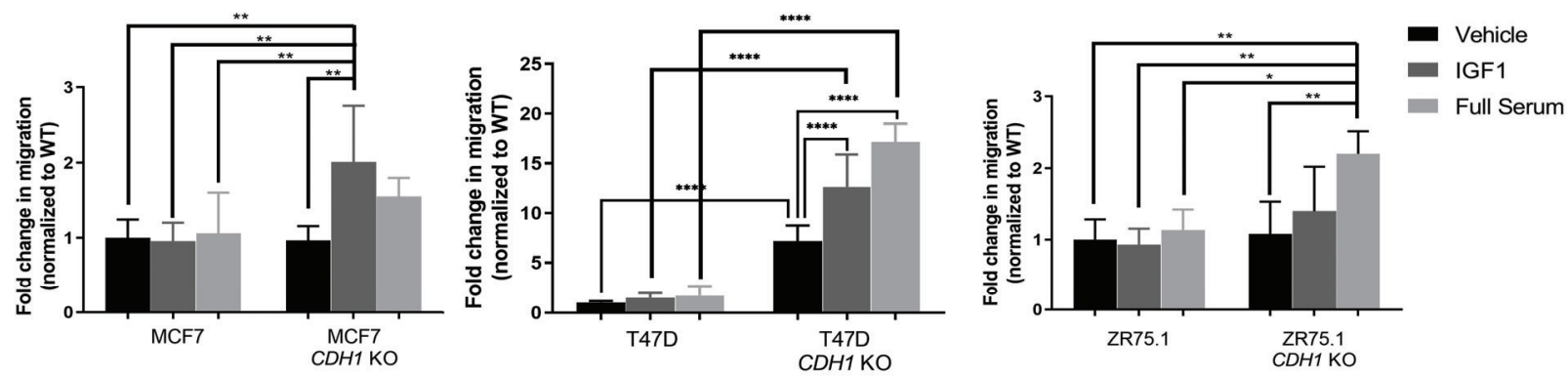

D

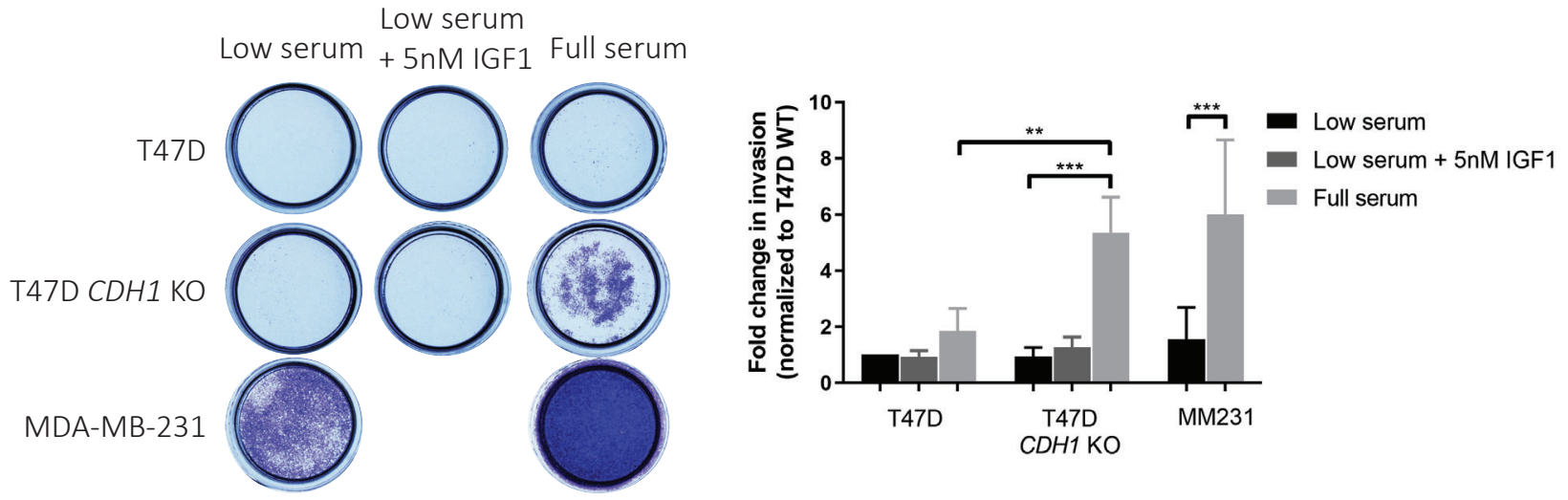

Figure 6: Loss of E-cadherin increases collagen I haptotaxis, IGF1-driven migration and serum-driven collagen invasion. (A) Images and quantification of Crystal Violet-stained Collagen I coated inserts from haptotaxis assays after 72 hours. Migrated cell colonies were quantified with ImageJ and plotted. (B) Images and (C) quantification of Crystal Violet-stained Transwell inserts from migration assays towards the indicated attractants after 72 hours. (D) Images and quantification of Crystal Violet-stained Collagen I inserts from invasion assays towards the indicated attractants after 72 hours. Graphs show representative data normalized to low serum WT cell samples from two to three independent experiments ( $\mathrm{N}=2$ biological replicates). $\mathrm{p}$-values from one-way ANOVA statistical testing. ${ }^{*} \mathrm{p} \leq 0.05$; ${ }^{* *} \mathrm{p} \leq$ $0.01 ; * * \mathrm{p} \leq 0.001 ; * * * * \mathrm{p} \leq 0.0001$. 
A

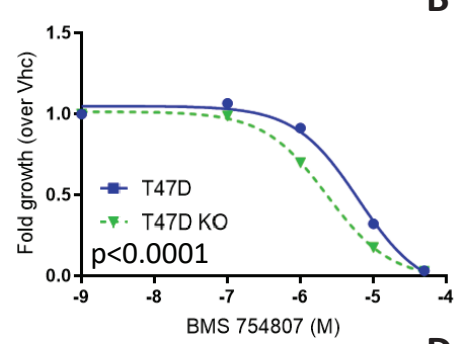

C

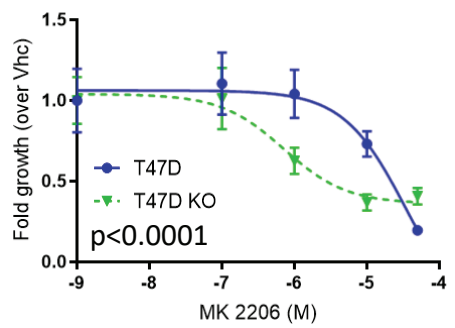

B

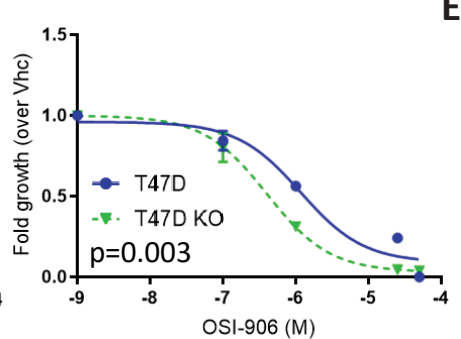

D

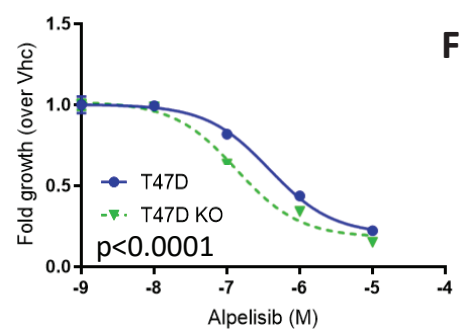

E

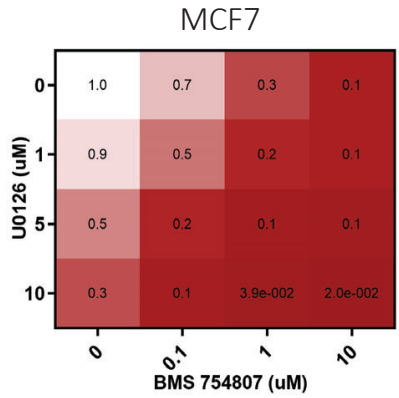

T47D

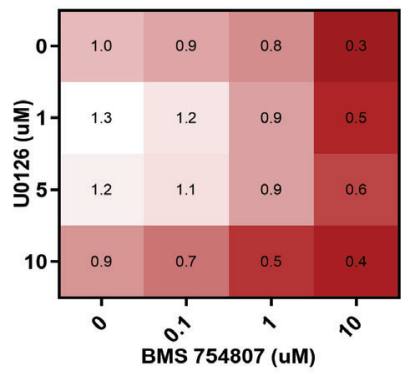

T47D

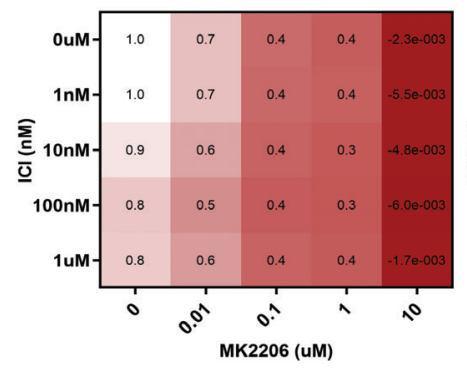

MCF7 CDH1 KO

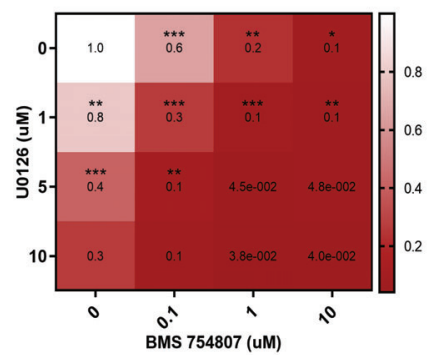

T47D CDH1 KO

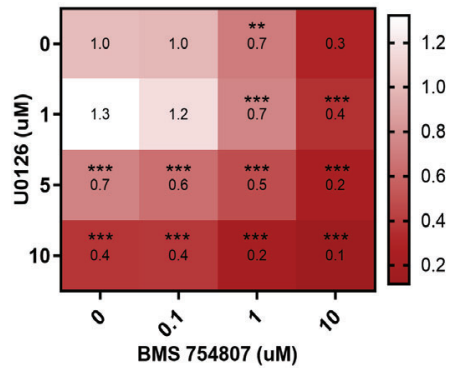

T47D CDH1 KO

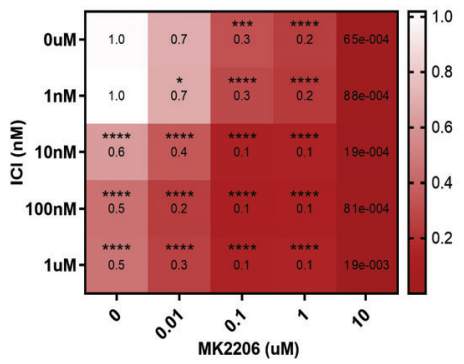

$2.62 \pm 1.27$

$0.368 \pm 0.22$

$0.539 \pm 0.18$

$0.239 \pm 0.11$

H

ILC

IDC

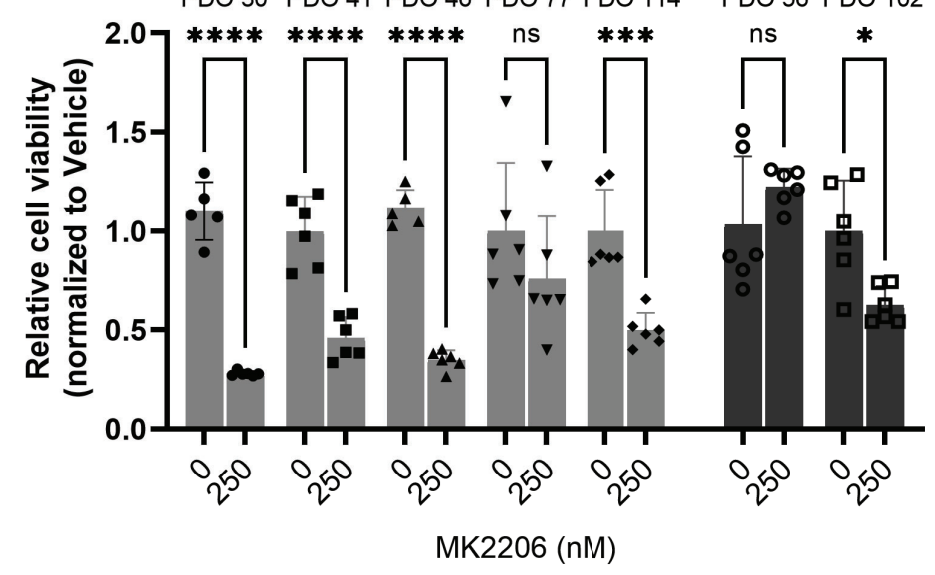

Figure 7: Loss of E-cadherin sensitizes cells to Fulvestrant and IGF1R/PI3K/Akt/MEK inhibitors in a context dependent manner. T47D parental and CDH1 KO cells were seeded in 96-well 2D plates and treated with IGF1R inhibitors (OSI-906 or BMS-754807), PI3K inhibitor (Alpelisib) or Akt inhibitor (MK2206) for 6 days. Conditions in the panels as follows: (A) BMS-754807; (B) OSI-906; (C) MK2206 and (D) Alpelisib. CellTiter Glo assay was used to assess cell viability (relative luminescence) and data was normalized to vehicle treated control. (E, F) Cells were treated a combination of MEK inhibitor (U0126) and BMS-754807 for 6 days and viability assessed as above. (G) Cells were treated a combination of Akt inhibitor (MK2206) and Fulvestrant for 6 days and viability assessed as above. (H) Patient derived IDC and ILC organoids were treated with Akt inhibitor (MK2206) for 12 days and viability assessed with CellTiter Glo 3D (relative luminescence), and data was normalized to vehicle treated control. IC50 values for viability were calculated by nonlinear regression and statistical differences evaluated using sum-of-squares Global f-test $(\mathrm{p}<0.05$; representative experiment shown; $\mathrm{N}=3$ (each with six biological replicates)). 
A

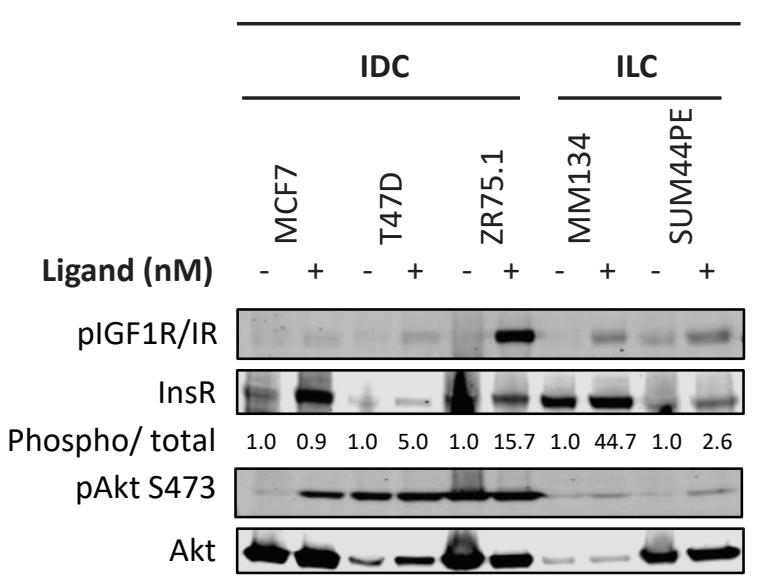

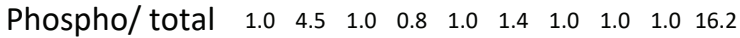

E-cadherin

$\beta$-actin
B

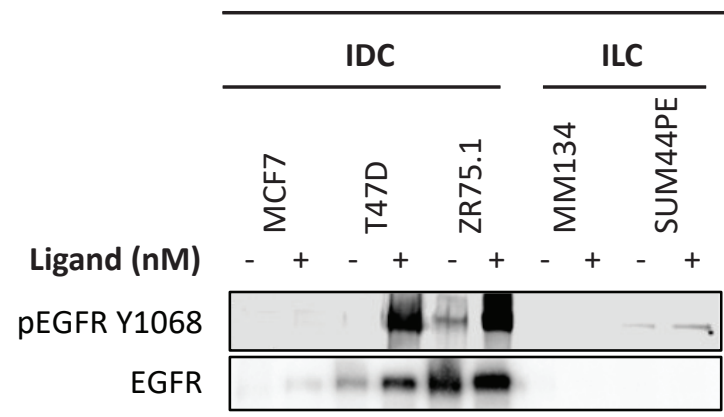

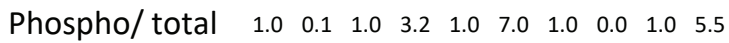

pAkt 5473

Akt

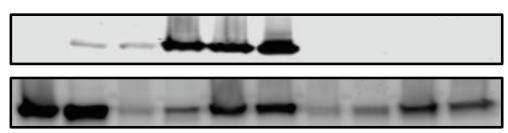

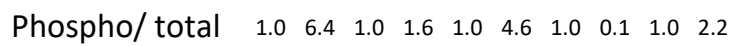

E-cadherin - - -

$\beta$-actin

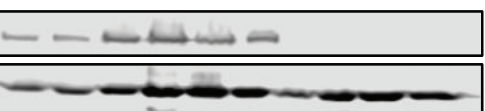

C

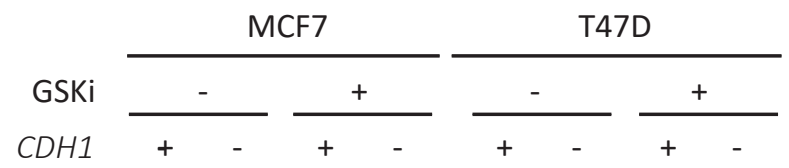

Non-phospho $\beta$ catenin (Ser33/37)

$\beta$-catenin

E-cadherin

$\beta$-actin

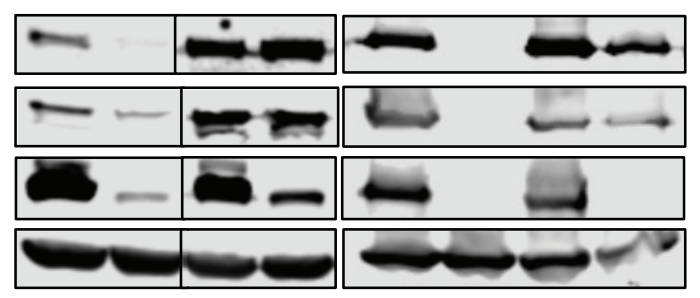

Supplementary Figure 1. (A) IDC cells (MCF7, T47D, ZR75.1) and ILC cells (MDA-MB-134-VI (MM134) and SUM44PE) were stimulated with doses of insulin or (B) EGF (0-5nM) for 15 minutes following an overnight serum starvation. IGF1R/IR, EGFR and Akt signaling was assessed by Western blotting. Phosphorylated protein levels were normalized over the corresponding total protein levels and loading control $\beta$-actin. Ligand treated samples values were further normalized to respective cell line's vehicle samples. (C) Cells were treated with GSK-3 inhibitor, CHIR 99021 for 24 hours and harvested for Western blotting. Total and active $\beta$-catenin protein levels were assessed. Representative experiment shown for all, $n=2-3$ for each experiment). 
A

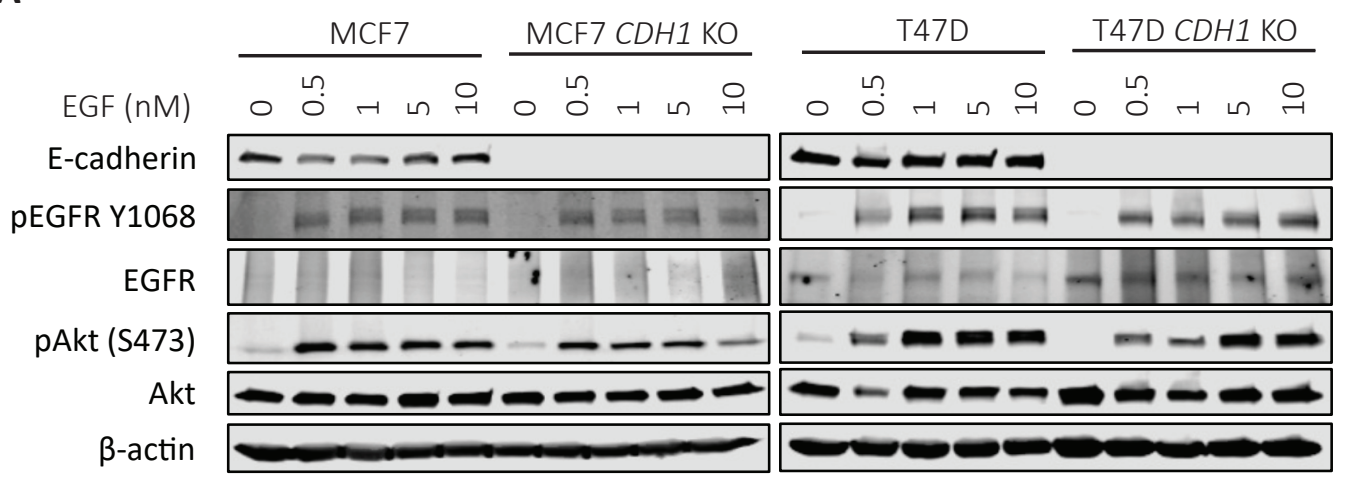

B

\begin{tabular}{|c|c|c|c|c|}
\hline & MCF7 & $\begin{array}{c}\text { MCF7 } \\
\text { CDH1 KO }\end{array}$ & T47D & $\begin{array}{c}\text { T47D } \\
\text { CDH1 KO }\end{array}$ \\
\hline ail & 30 & 光 & $3 \frac{c}{300}$ & 芳 \\
\hline
\end{tabular}

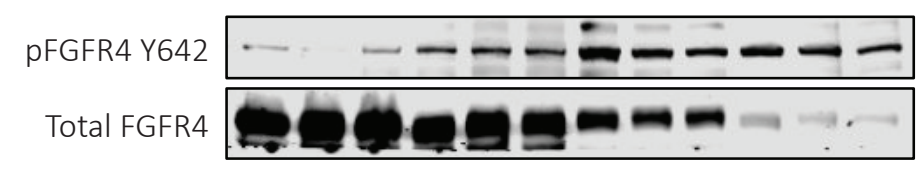

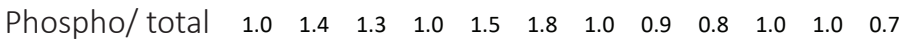

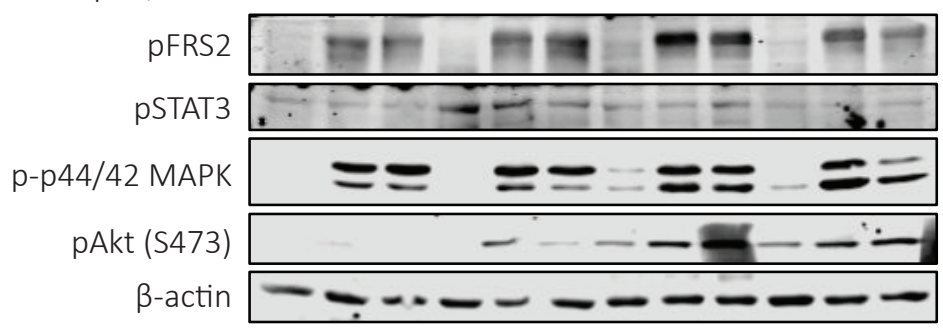

ZR75.1 ZR75.1 CDH1 KO

Treatment duration

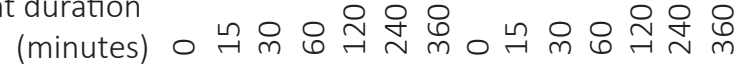

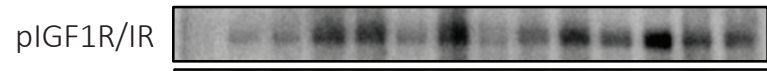

Total IGF1R 1 -

pAkt (S473) «- - - - - - - -

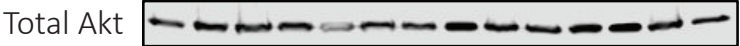

E-cadherin $-\ldots-\cdots-\cdots$

$\beta$-actin

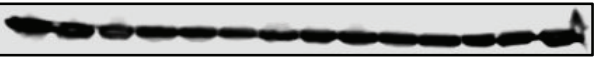

Supplementary Figure 2. Cells were serum starved overnight and stimulated with (A) EGF (0-10nM) and (B) cocktail of FGF ligands $(10-50 \mathrm{ng} / \mathrm{mL}$ ) for 15 minutes. Cells were harvested for Western blot to assess downstream signaling. (C) ZR75.1 parental and CDH1 KO cells were treated with 10nM IGF1 for a time course from 0-6 hours to assess the duration of signaling activity. Representative experiment shown for all, $n=2-3$ for each experiment). 


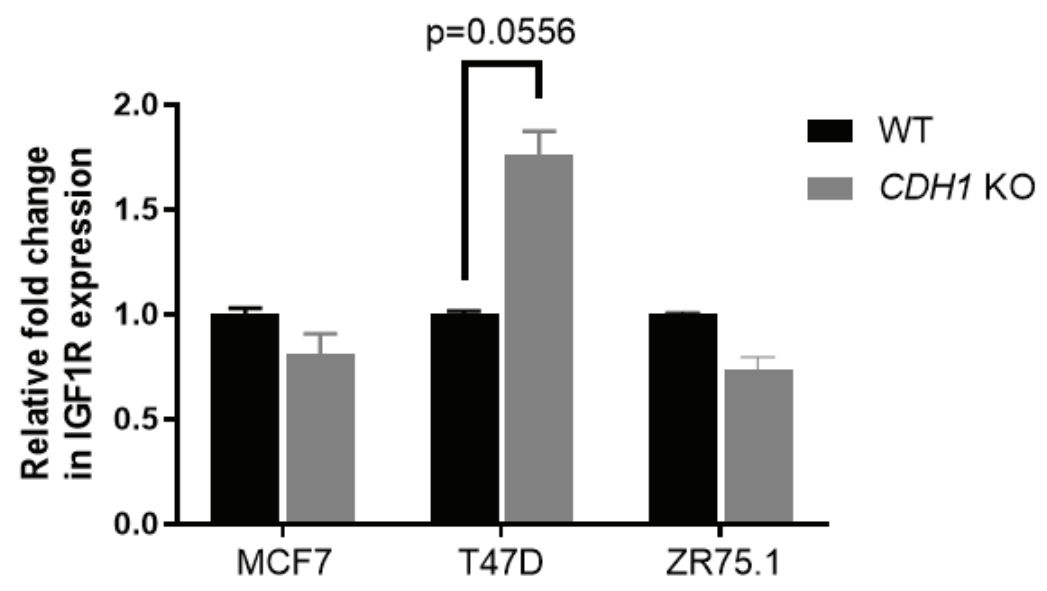

Supplementary Figure 3. (A) qRT-PCR for IGF1R for the cell line models studied. Fold change in expression were calculated by normalizing delta Ct values to the respective WT values. Statistical differences were evaluated using paired t-test ( $p=0.0556$ for T47D cell pair). Representative experiment shown, $n=2$ experiments with 2 biological and 3 technical repeats for each cell line. 
A

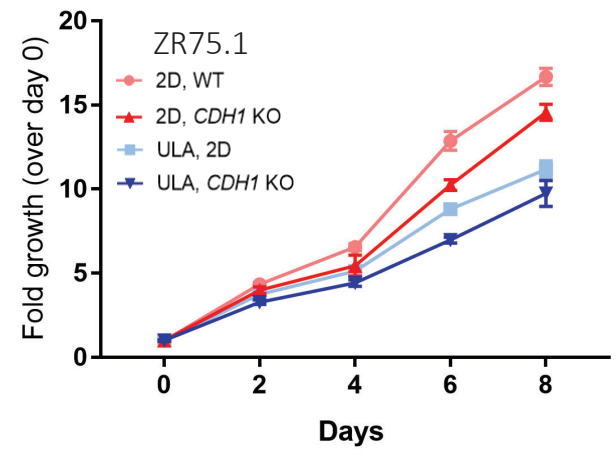

C

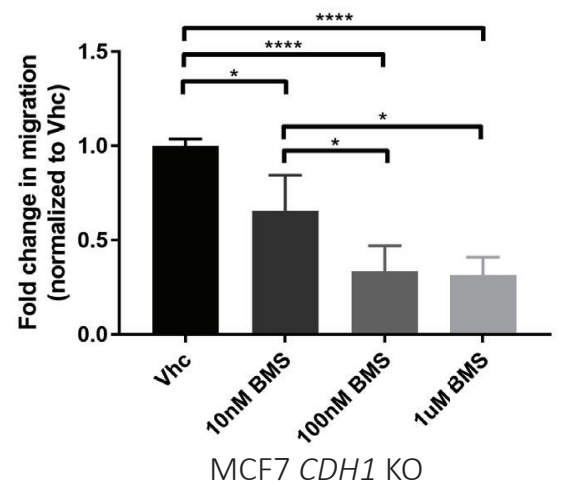

D

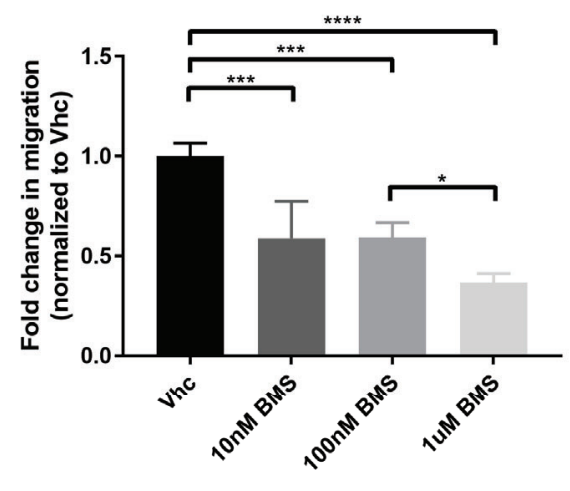

B

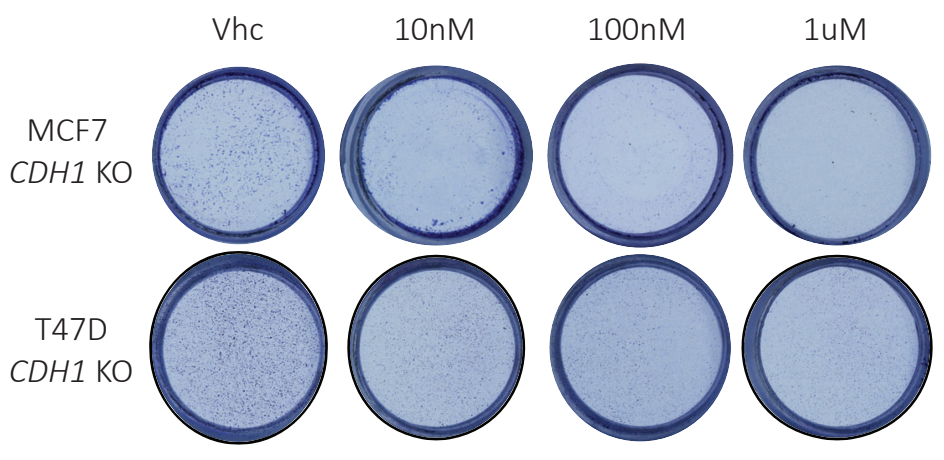

E

MCF7 $\mathrm{CDH} 1 \mathrm{KO}$ MCF7 Low serum
Low serum
$+5 n M$ IGF1

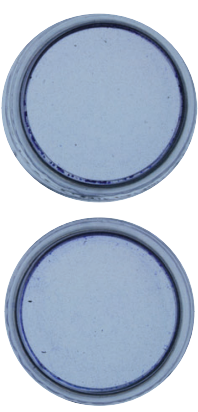

ZR75.1

ZR75.1

$\mathrm{CDH} 1 \mathrm{KO}$

Full serum

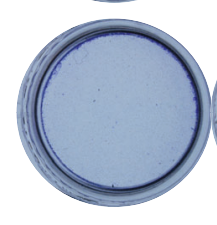

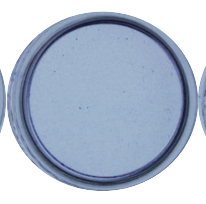
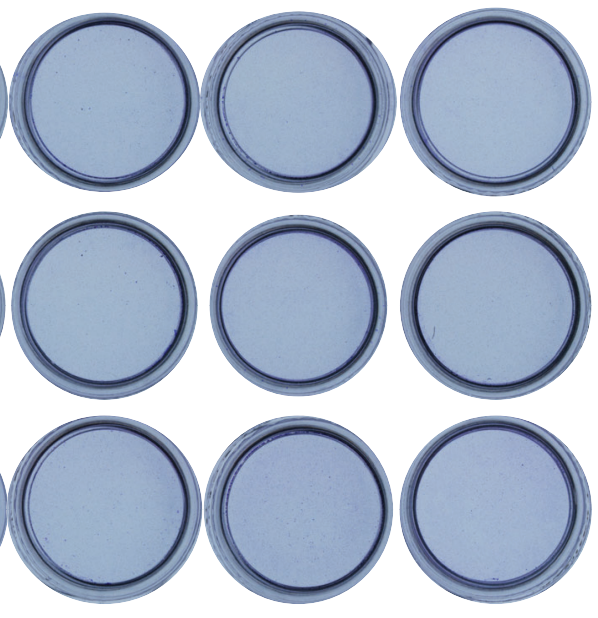

T47D CDH1 KO

Supplementary Figure 4. (A) Representative 2D and ULA growth of ZR75.1 parental and CDH1 KO cells quantified by CellTiter-Glo and normalized to Day 0. (B) Images and (C,D) quantification of crystal violet-stained Transwell inserts from migration assays towards low serum + 5nM IGF1 media after 72 hours. Cells were treated with increasing doses of BMS-754807 in the upper chamber to assess IGF1 specific migration. Graphs show representative data normalized to vehicle from two to three independent experiments ( $n=2$ biological replicates). $p$-values are from one-way ANOVA. ${ }^{*} p \leq 0.05 ;{ }^{* *} p \leq 0.01 ; * * p \leq 0.001 ; * * * * \leq 0.0001$. (E) Images of crystal violet-stained collagen I inserts from invasion assays towards the indicated attractants after 72 hours. 
A
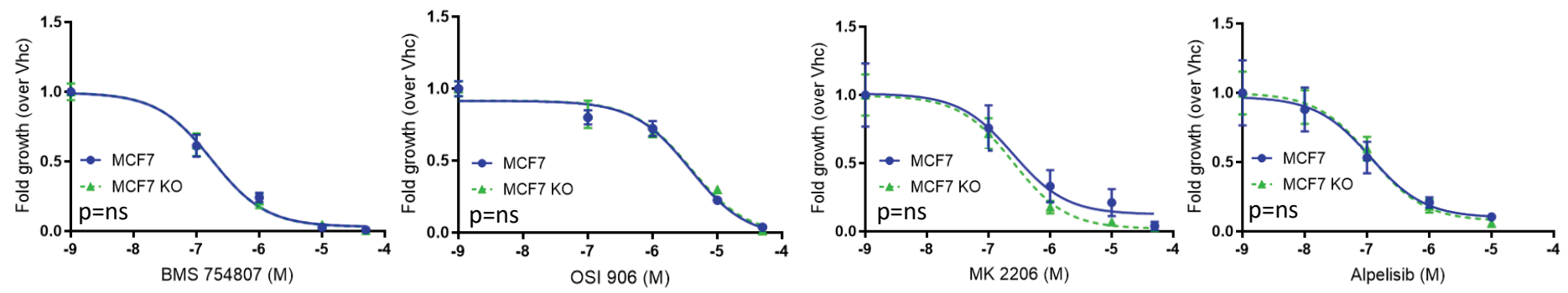

B
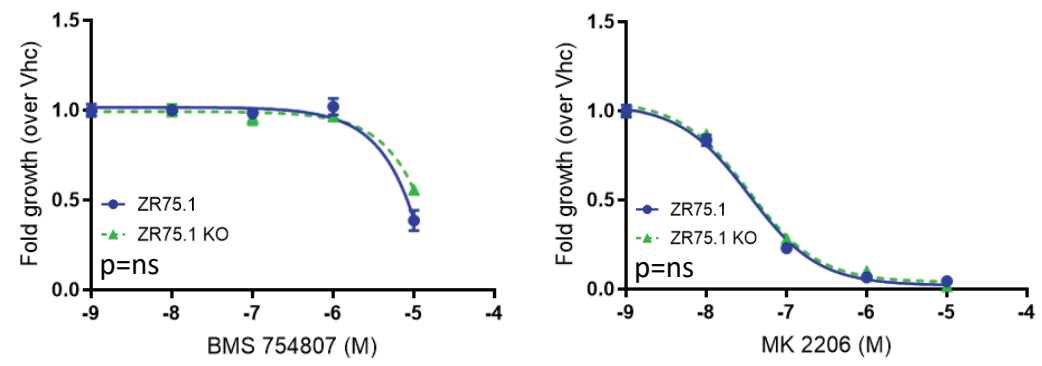

C
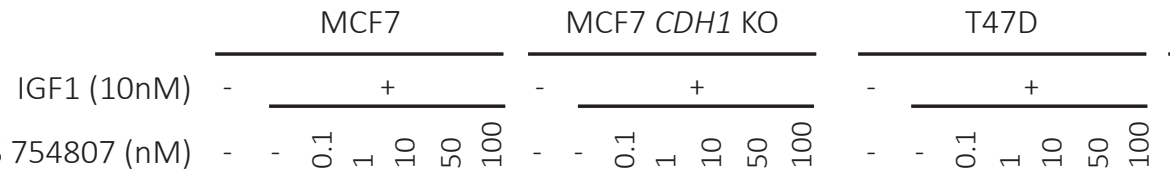

T47D CDH1 KO
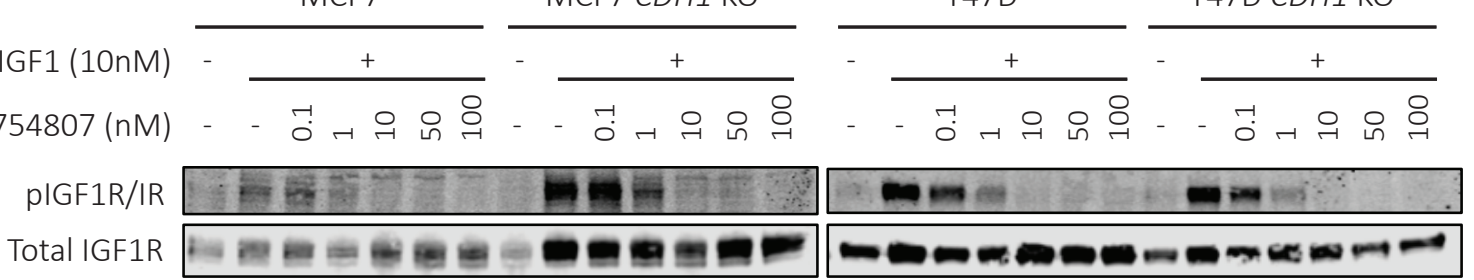

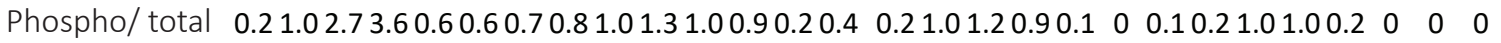

pAkt (S473)
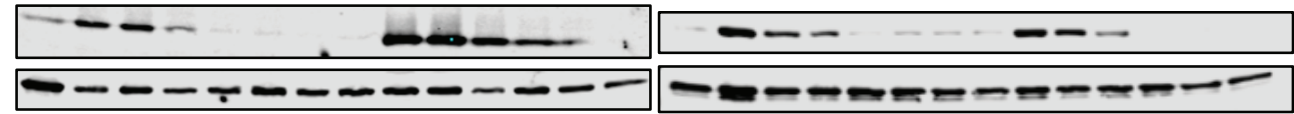

Phospho/ total $0 \begin{array}{lllllllll}0 & 1.00 .50 .5 & 0 & 0 & 0 & 0 & 1.01 .01 .20 .20 .10 .1 & 0.31 .00 .70 .50 .10 .20 .20 .21 .00 .90 .40 .10 .20 .1\end{array}$

$$
\text { E-cadherin }
$$

$\beta$-actin

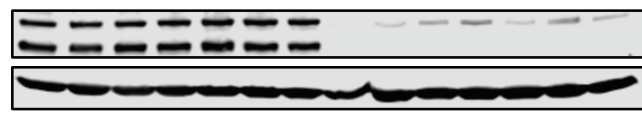

D

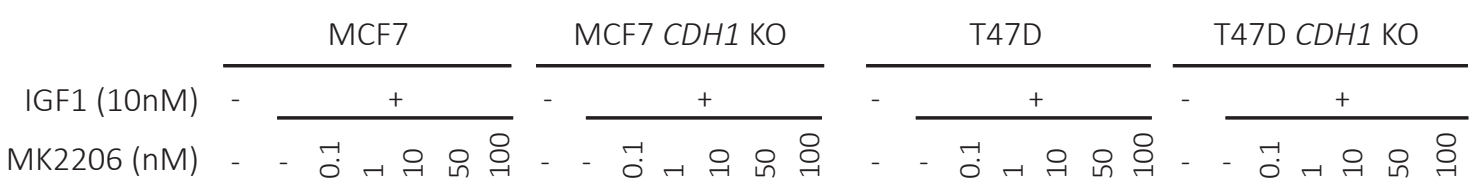

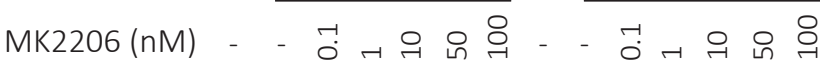

pIGF1R/IR

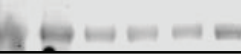

$\mathrm{a}=\mathrm{a}=\mathrm{m}$

Total IGF1R
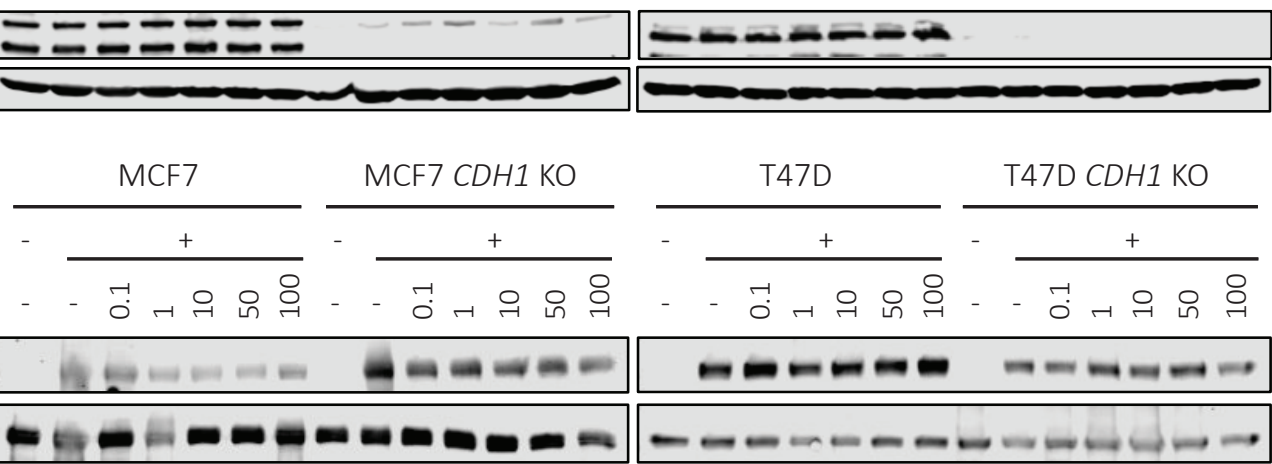

Phospho/ total 0 1.0 0.61.20.40.50.6 0 1.0 0.5 0.4 0.4 0.50.5

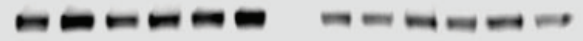
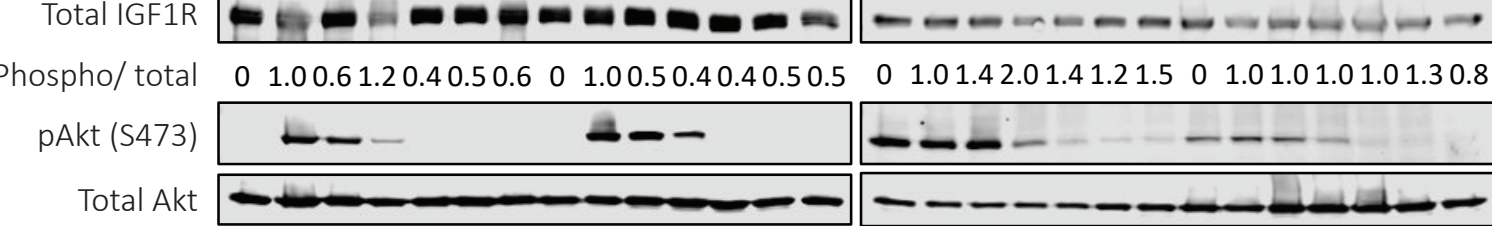

Phospho/ total $0 \begin{array}{lllllllllllll}0 & 1.0 & 0.10 .1 & 0 & 0 & 0 & 0 & 1.00 .7 & 0.2 & 0 & 0 & 0\end{array}$

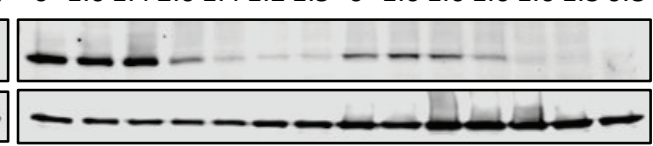

$\begin{array}{rrrrr}1.00 .10 .1 & 0 & 0 & 0\end{array}$

$0.71 .01 .10 .30 .1 \quad 0 \quad 0 \quad 0.71 .00 .50 .30 .10 .10 .1$

E-cadherin

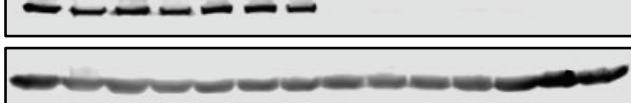

$-\infty-\infty-\infty$

$\beta$-actin

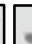

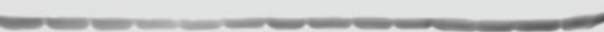

Supplementary Figure 5. (A) MCF7 parental and CDH1 KO cells and (B) ZR75.1 parental and CDH1 KO cells were treated with IGF1R inhibitor (OSI-906 or BMS-754807) or PI3K inhibitor (Alpelisib) or Akt inhibitor (MK2206) for 6 days. CellTiter Glo assay was used to assess cell viability (relative luminescence). IC50 values for viability were calculated by nonlinear regression and statistical differences evaluated using sum-of-squares Global f-test ( $P<0.05$; representative experiment shown; $n=3$ each with six biological replicates). Cells were treated with (C) BMS754807 and (D) MK2206 in increasing doses to assess signaling inhibition of the compounds used for cell viability assays. Representative experiment shown, $\mathrm{n}=2$ for each experiment). 
Supplemeintall-Pigprint doi: https://doi.org/10.1101/2022.02.22.480917; this version posted February 22, 2022. The copyright holder for this preprint

A

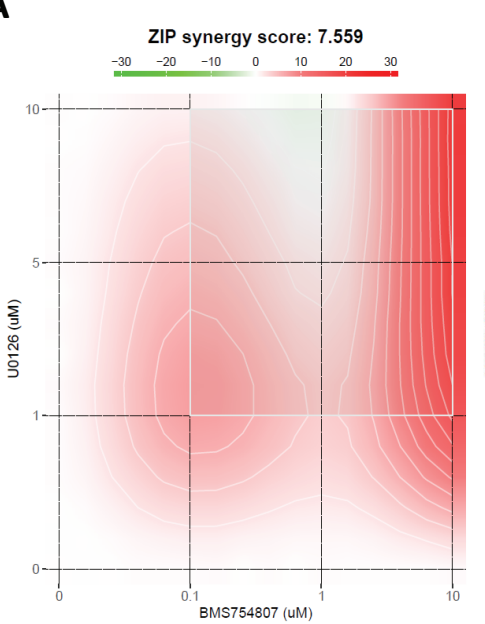

MCF7

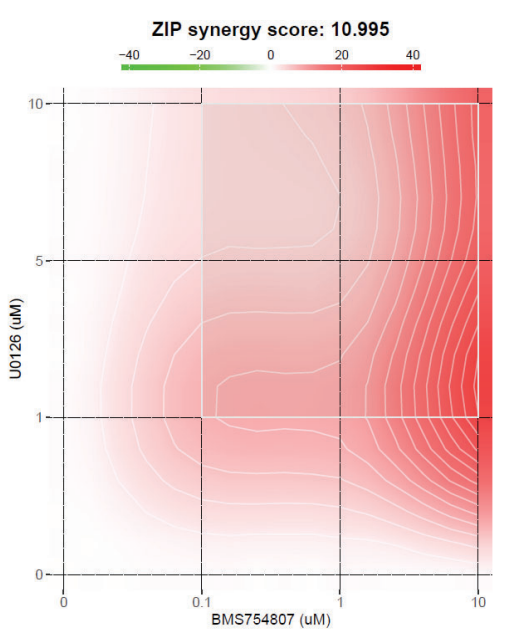

MCF7 CDH1 KO
B

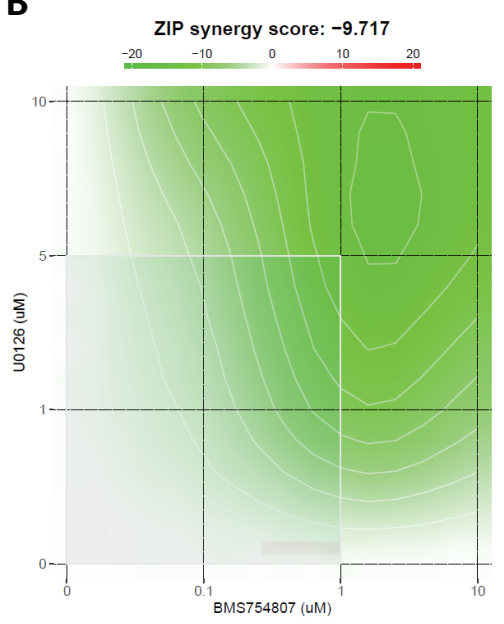

T47D

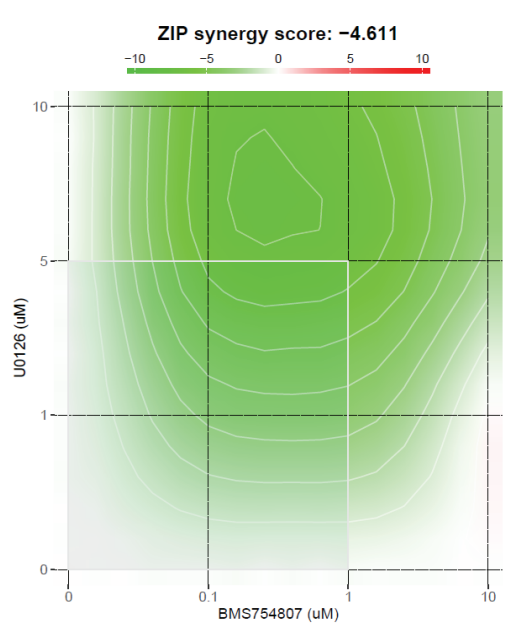

T47D CDH1 KO

C

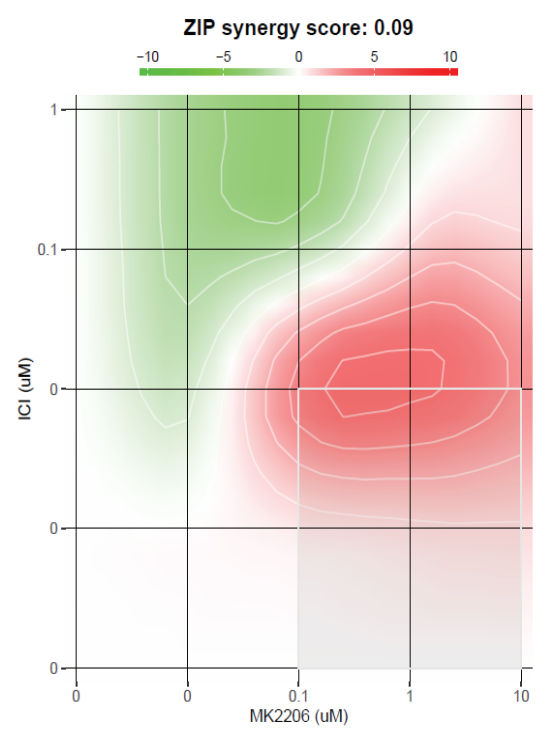

T47D

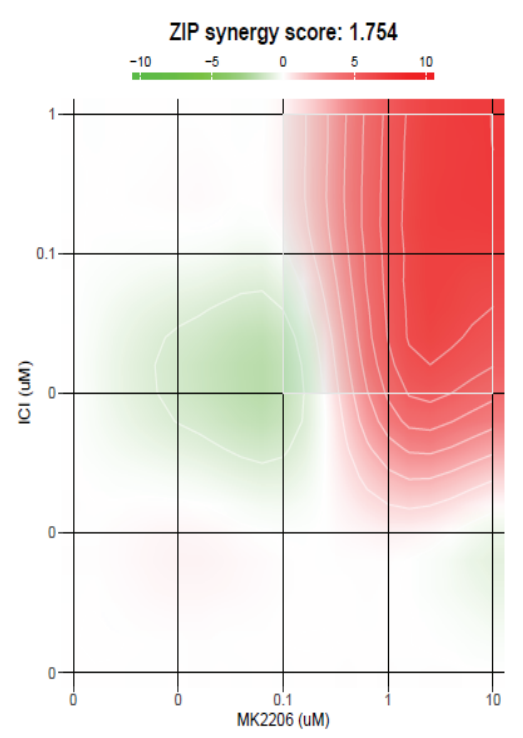

T47D CDH1 KO

D

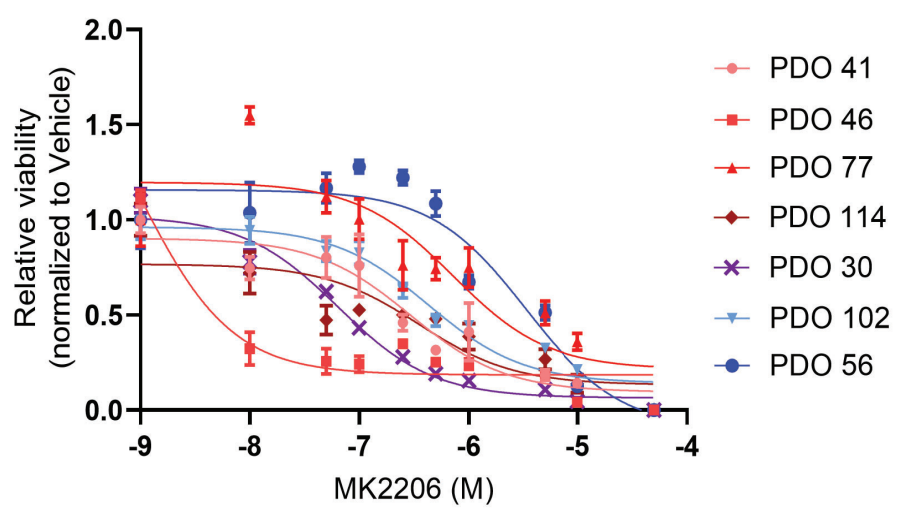

Supplementary Figure 6. Results from (A) MCF7 WT and CDH1 KO cells and (B) T47D WT and CDH1 KO cells were treated with a combination of IGF1R inhibitor (BMS-754807) and MEK inhibitor (U0126) for 6 days. (C) T47D WT and CDH1 KO cells were treated with a combination of a SERD (Fulvestrant) and Akt inhibitor (MK2206) for 6 days. CellTiter Glo assay was used to assess cell viability (relative luminescence). The viability values were input into the SynergyFinder platform and assessed for drug synergy effect. Synergy scores -10 to 10 signify an additive effect of the compounds being tested. (D) Complete dose response analysis of ILC and ILC organoids assayed for sensitivity to MK2206. Organoids were plated and treated in 96-well for 12 days. CellTiter Glo 3D assay was used to assess cell viability (relative luminescence) and data normalized to vehicle treated control. Representative experiment shown for all, $n=2-3$ for each experiment). 